\title{
ANALYTIC TORSION FOR GROUP ACTIONS
}

\author{
JOHN LOTT \& MEL ROTHENBERG
}

\section{Introduction}

The Reidemeister torsion is a classical topological invariant for nonsimply-connected manifolds [14]. Let $M$ be a closed oriented smooth manifold with the fundamental group $\pi_{1}(M)$ nontrivial. Let $\rho: \pi_{1}(M) \rightarrow$ $\mathrm{O}(N)$ be an orthogonal representation of $\pi_{1}(M)$. If the twisted real cohomology $H^{*}\left(M, E_{\rho}\right)$ vanishes, then one can define the Reidemeister torsion $\tau_{\rho} \in \mathbb{R}$, which is a homeomorphism invariant of $M$. The original interest of $\tau_{\rho}$ was that it is not a homotopy invariant, and so can distinguish spaces which are homotopy equivalent but are not homeomorphic.

Ray and Singer asked whether, as for many other topological quantities, one can compute $\tau_{\rho}$ by analytic methods [17]. Given a Riemannian metric $g$ on $M$, they defined an analytic torsion $T_{\rho} \in \mathbb{R}$ as a certain combination of the eigenvalues of the Laplacian acting on twisted differential forms. They showed that under the above acyclicity condition, $T_{\rho}$ is independent of the metric $g$, and they conjectured that the analytic expression $T_{\rho}$ equals the combinatorial expression $\tau_{\rho}$. This conjecture was proven to be true independently by Cheeger [4] and Müller [15].

One can look at the above situation in the following way. The group $\pi_{1}(M)$ acts freely on the universal cover $\widetilde{M}$, and so one has an invariant for free group actions. A natural question is whether the Reidemeister torsion can be extended to an invariant for more general group actions. For a finite group acting (not necessarily freely) on a closed oriented PL manifold $X$, a Reidemeister torsion was defined algebraically by Rothenberg [19]. One can then ask whether there is a corresponding analytic torsion when $X$ is smooth, and whether the analytic torsion equals the combinatorial torsion.

In $\S$ II we define the analytic torsion $T_{\rho}$ for a finite group action and show that if the relevant cohomology groups vanish, then it is independent of the $G$-invariant metric used in its definition. (This was shown previously in unpublished work by Cheeger [5].) The analysis involved to

Received June 11, 1990. 
prove this is similar to that of the analogous statement for the ordinary analytic torsion, in that it involves a heat kernel analysis, but differs in that it is necessary to use the heat kernel asymptotics off of the diagonal. We prove some product formulas for $T_{\rho}$ in $\S \mathrm{III}$, and compute $T_{\rho}$ for special orthogonal actions on spheres in $\S I V$. We define the combinatorial torsion $\tau_{\rho}$, based on [19], in $\S \mathrm{V}$, and compute $\tau_{\rho}$ for special orthogonal actions on spheres in $\S \mathrm{VI}$.

We were surprised to find that, in general, $T_{\rho}$ does not equal $\tau_{\rho}$. One can see this for special orthogonal actions on spheres. If the sphere is odd-dimensional then $T_{\rho}=\tau_{\rho}$, but if the sphere is even-dimensional then $T_{\rho}$ vanishes, whereas $\tau_{\rho}$ generally does not. In $\S$ VII we show that $T_{\rho}$ and $\tau_{\rho}$ coincide for all orientation-preserving finite group actions on odddimensional spaces. As in the case of the ordinary analytic torsion, the proof is done using surgery arguments, now generalized to the equivariant case.

In $\S$ VIII we discuss the case of even-dimensional spaces. If the space $X$ is even-dimensional, then $T_{\rho}$ vanishes from an argument involving the Hodge duality operator. If the action is free, then $\tau_{\rho}$ also vanishes. However, if the action is not free, then one finds that the corresponding combinatorial arguments, involving the dual cell complex, fail.

From the argument of the proof in $\S$ VII, we do derive an expression that tells how $\tau_{\rho}$ changes under equivariant surgery in the even-dimensional case. Although we first found this expression by analytic means, we later found a purely topological proof, which we also present.

It is well known that the Reidemeister torsion has many similarities with the Euler characteristic (see [8] for a exposition from this point of view). In $\S$ VIII, for the even-dimensional case, we give an expression for $\tau_{\rho}$ in terms of a Morse function on $X$, and also an expression for $\tau_{\rho}$ in terms of an excision of a subspace of maximal isotropy group. Both of these relations show a striking resemblance to the corresponding equations for the Euler characteristic. They make the combinatorial torsion very computable in the even-dimensional case. For example, we use them to show that for a $\mathbb{Z}_{p}$ action, the exponential of twice the torsion is the absolute value of an element of a certain multiplicative subgroup of $\mathbb{Q}(\exp (2 \pi i / p))$. In $\S \mathrm{IX}$ we consider the torsion for orientation-reversing group actions. We show that the analytic and combinatorial torsions agree for an orientation-preserving group action in odd dimensions, or an orientation-reversing group action in even dimensions. We use this to derive some results on the torsion of a manifold with boundary by looking at the involution on the double of the manifold. 
One can ask whether one can use the analytic methods to define invariants for group actions without usual combinatorial invariants. In $\S \mathrm{X}$ we define the analytic torsion for discrete group actions and study its properties. In $\S \mathrm{XI}$ we give an example of its application in proving by purely analytic means (in the odd-dimensional case) a theorem of de Rham, stating that orthogonal group actions on spheres are smoothly equivalent if and only if they are linearly equivalent.

An exposition of the results of this paper appeared in [12].

We would like to thank David Fried and Isadore Singer for helpful discussions. We would like to thank Wolfgang Lück for discussions and for pointing out a gap in our original proof of Proposition 16. He has independently derived results for the combinatorial torsion for manifolds with boundary which are analogous to those of §IX [13]. We would like to thank Jeff Cheeger for informing us of his unpublished results. One of us (J.L.) would like to thank M. Berger and the IHES for their hospitality while part of this research was performed.

\section{The analytic torsion of finite group actions}

Let us recall the definition of the ordinary analytic torsion. Let $M$ be an $n$-dimensional closed smooth oriented Riemannian manifold with metric $g$. Let $\rho: \pi_{1}(M) \rightarrow \mathrm{O}(N)$ be an orthogonal representation of the fundamental group of $M$, which then gives a flat $\mathbb{R}^{N}$ bundle $E_{\rho}$ over $M$. One has the de Rham complex

$$
\Lambda^{0}\left(M, E_{\rho}\right) \stackrel{d}{\longrightarrow} \Lambda^{1}\left(M, E_{\rho}\right) \stackrel{d}{\longrightarrow} \cdots \stackrel{d}{\longrightarrow} \Lambda^{n}\left(M, E_{\rho}\right)
$$

of smooth differential forms on $M$ with value in $E_{\rho}$. Using the metric one has an inner product on $\Lambda^{k}\left(M, E_{\rho}\right)$ and so one has the adjoint operator

$$
\delta: \Lambda^{k}\left(M, E_{\rho}\right) \rightarrow \Lambda^{k-1}\left(M, E_{\rho}\right)
$$

Let $\Delta_{k}$ denote the Laplacian $d \delta+\delta d$ acting on $\Lambda^{k}\left(M, E_{\rho}\right)$. The kernel of $\Delta_{k}$ is isomorphic to the real cohomology group $H^{k}\left(M, E_{\rho}\right)$, which inherits an inner product from that of $\Lambda^{k}\left(M, E_{\rho}\right)$. Let $P_{k}$ denote the projection of the Hilbert space $\mathscr{H}^{k}\left(M, E_{\rho}\right)$ of $L^{2} k$-forms onto $\operatorname{Ker} \Delta_{k}$. Let $\Delta_{k}^{\prime}$ denote $\Delta_{k}$ acting on $\operatorname{Im} \Delta_{k}$. By abuse of notation, we will write $\exp \left(-T \Delta_{k}^{\prime}\right)$ to denote $\exp \left(-T \Delta_{k}\right)-P_{k}$, acting on $\mathscr{H}^{k}\left(M, E_{\rho}\right)$. Let $F$ 
denote the operator on $\bigoplus_{k} \mathscr{H}^{k}\left(M, E_{\rho}\right)$, which is multiplication by $k$ on $\mathscr{H}^{k}\left(M, E_{\rho}\right)$, and let $(-)^{F}$ denote the operator which is multiplication by $(-1)^{k}$ on $\mathscr{H}^{k}\left(M, E_{\rho}\right)$.

Definition. The ordinary analytic torsion $\widetilde{T}_{\rho}$ is given by

$$
\widetilde{T}_{\rho}=\left.\frac{d}{d s}\right|_{s=0} \frac{1}{\Gamma(s)} \int_{0}^{\infty} T^{s-1} \operatorname{Tr}(-)^{F} F e^{-T \Delta^{\prime}} d T,
$$

where $\operatorname{Tr}$ denotes the trace of a trace class operator acting on $\bigoplus_{k} \mathscr{H}^{k}\left(M, E_{\rho}\right)$.

(It is understood that the derivative at $s=0$ is that of a meromorphic function in $s$, which is defined by analytic continuation from $\operatorname{Re} s \gg 0$, and which is analytic around $s=0$.)

The remarkable fact about this expression is that if $\bigoplus_{k} H^{k}\left(M, E_{\rho}\right)$ vanishes, then $\widetilde{T}_{\rho}$ is independent of the metric used in the definition and equals the combinatorial Reidemeister torsion [4], [15].

One can think of the analytic torsion as being an invariant for a nonsimply-connected manifold, or, equivalently, as an invariant for the free action of $\pi_{1}(M)$ on the universal covering space $\widetilde{M}$. In the special case when $\widetilde{M}$ is compact, let

$$
a: \pi_{1}(M) \rightarrow \operatorname{Diff}(\widetilde{M})
$$

denote the covering transformations. If $g$ is an element of $\pi_{1}(M)$, let $a\left(g^{-1}\right)^{*}$ denote the action of $g$ on the differential forms on $\widetilde{M}$ :

$$
a\left(g^{-1}\right)^{*}: \bigoplus_{k} \mathscr{H}^{k}(\widetilde{M}) \rightarrow \bigoplus_{k} \mathscr{H}^{k}(\widetilde{M})
$$

Consider the projection operator

$$
\Pi=\frac{1}{\left|\pi_{1}(M)\right|} \sum_{g \in \pi_{1}(M)} \rho(g) a\left(g^{-1}\right)^{*}
$$

acting on $\bigoplus_{k}\left(\mathscr{H}^{k}(\widetilde{M}) \otimes \mathbb{R}^{N}\right)$, whose range is $\bigoplus_{k} \mathscr{H}^{k}\left(M, E_{\rho}\right)$. Then we can write

$$
\widetilde{T}_{\rho}=\left.\frac{d}{d s}\right|_{s=0} \frac{1}{\Gamma(s)} \int_{0}^{\infty} T^{s-1} \operatorname{Tr}(-)^{F} F \Pi e^{-T \Delta^{\prime}} d T,
$$

where the $\operatorname{Tr}$ now denotes the trace of a trace class operator acting on $\bigoplus_{k}\left(\mathscr{H}^{k}(\widetilde{M}) \otimes \mathbb{R}^{N}\right)$.

These considerations motivate the following generalization. Let $G$ be a finite group acting smoothly by orientation-preserving diffeomorphisms 
on a closed oriented smooth $n$-dimensional Riemannian manifold $X$, equipped with a $G$-invariant metric $g$. Let $\rho: G \rightarrow \mathrm{O}(N)$ be an orthogonal representation of $G$. Put

$$
\Pi=\frac{1}{|G|} \sum_{g \in G} \rho(g) a\left(g^{-1}\right)^{*},
$$

a projection operator acting on $\bigoplus_{k}\left(\mathscr{H}^{k}(X) \otimes \mathbb{R}^{N}\right)$.

Definition.

$$
T_{\rho}=\left.\frac{d}{d s}\right|_{s=0} \frac{1}{\Gamma(s)} \int_{0}^{\infty} T^{s-1} \operatorname{Tr}(-)^{F} F \Pi e^{-T \Delta^{\prime}} d T,
$$

where $\operatorname{Tr}$ now denotes the trace of a trace class operator acting on $\bigoplus_{k}\left(\mathscr{H}^{k}(X) \otimes \mathbb{R}^{N}\right)$.

(From the estimates in the proof of Proposition 2 it will follow that we are taking the derivative at $s=0$ of a meromorphic function which is defined by analytic continuation from $\operatorname{Re} s \gg 0$, and is analytic around $s=0$.)

We will now prove two properties of $T_{\rho}$, which are analogous to those of the ordinary analytic torsion [17].

Consider the $d$-operator, acting on the $\rho$-equivariant $\mathbb{R}^{N}$-valued differential forms, that is, on $\Pi\left(\bigoplus_{k}\left(\mathscr{C}^{k}(X) \otimes \mathbb{R}^{N}\right)\right)$. Let $\bigoplus_{k} H^{k}(X, \rho)$ denote the cohomology of this complex, with the induced inner product structure. Let $*$ denote the Hodge duality operator.

Proposition 1. If $n$ is even, then $T_{\rho}=0$.

Proof. The operator $*$ commutes with $\Delta^{\prime}$ and $a\left(g^{-1}\right)^{*}$, and satisfies

$$
F *+* F=n *, \quad(-)^{F} *=(-)^{n} *(-)^{F} .
$$

Thus

$$
\begin{aligned}
\operatorname{Tr}(-)^{F} F a\left(g^{-1}\right)^{*} e^{-T \Delta^{\prime}} & =\operatorname{Tr}(-)^{F} F a\left(g^{-1}\right)^{*} e^{-T \Delta^{\prime}} * *^{-1} \\
& =\operatorname{Tr} *^{-1}(-)^{F} F * a\left(g^{-1}\right)^{*} e^{-T \Delta^{\prime}} \\
& =(-)^{n} \operatorname{Tr}(-)^{F}(n-F) a\left(g^{-1}\right)^{*} e^{-T \Delta^{\prime}} .
\end{aligned}
$$

We can consider the above trace to be on trace class operators acting on $\operatorname{Im} \Delta$. Let us define the operator $Q$ on $\operatorname{Im} \Delta$ by

$$
Q=(d+\delta)\left(\Delta^{\prime}\right)^{-1 / 2} \text {. }
$$

Then

$$
Q^{2}=1 \text { and } Q(-)^{F}+(-)^{F} Q=0
$$


It follows that

$$
\begin{aligned}
\operatorname{Tr}(-)^{F} a\left(g^{-1}\right)^{*} e^{-T \Delta^{\prime}} & =\operatorname{Tr} Q^{2}(-)^{F} a\left(g^{-1}\right)^{*} e^{-T \Delta^{\prime}} \\
& =\operatorname{Tr} Q(-)^{F} a\left(g^{-1}\right)^{*} e^{-T \Delta^{\prime}} Q \\
& =-\operatorname{Tr} Q^{2}(-)^{F} a\left(g^{-1}\right)^{*} e^{-T \Delta^{\prime}}=0
\end{aligned}
$$

Then

$$
\operatorname{Tr}(-)^{F} F a\left(g^{-1}\right)^{*} e^{-T \Delta^{\prime}}=(-)^{n+1} \operatorname{Tr}(-)^{F} F a\left(g^{-1}\right)^{*} e^{-T \Delta^{\prime}}=0 .
$$

Thus the integrand for $T_{\rho}$ vanishes.

Proposition 2. If $n$ is odd, let $g(\varepsilon)$ be a smooth 1-parameter family of $G$-invariant metrics on $X$. Then

$$
\frac{d}{d \varepsilon} T_{\rho}=-\left.\operatorname{Tr}\right|_{\operatorname{Ker} \Delta} \Pi(-)^{F}\left(\frac{d}{d \varepsilon} *\right) *^{-1} .
$$

Proof. In the ensuing proof, we will make some manipulations which are initially only justified when $\operatorname{Re} s \gg 0$. Because everything will be analytically continued to $s=0$, it will follow that the manipulations are also justified around $s=0$.

Let $V$ denote $\left(\frac{d}{d \varepsilon} *\right) *^{-1}$. Because the $d$-operator is defined independently of the metric $\frac{d}{d \varepsilon} d=0$, but

$$
\frac{d}{d \varepsilon} \delta=\frac{d}{d \varepsilon}( \pm * d *)=[V, \delta]
$$

Thus

$$
\frac{d}{d \varepsilon} \Delta=\{d,[V, \delta]\}
$$

where $\{\bullet, \bullet\}$ denotes anticommutation. We also need that $a\left(g^{-1}\right)^{*}$ commutes with $*, d, \delta$, and $F$, and that

$$
[F, d]=d, \quad[F, \delta]=-\delta .
$$

Then

$$
\begin{aligned}
\frac{d}{d \varepsilon} T_{\rho}=\left.\frac{d}{d \varepsilon} \frac{d}{d s}\right|_{s=0} \frac{1}{\Gamma(s)} \int_{0}^{\infty} T^{s-1}\left[\operatorname{Tr}(-)^{F} F \Pi e^{-T \Delta}\right. & \left.-\left.\operatorname{Tr}\right|_{\operatorname{Ker} \Delta}(-)^{F} F \Pi\right] d T .
\end{aligned}
$$

Let $A$ denote the de Rham isomorphism from $\operatorname{Ker} \Delta \otimes \mathbb{R}^{N}$ to $H^{*}(X) \otimes$ $\mathbb{R}^{N}$. As $G$ acts by isometries on $X$, we have that

$$
A a\left(g^{-1}\right)^{*} A^{-1}=a\left(g^{-1}\right)^{*}
$$


where, on the right-hand side, $a\left(g^{-1}\right)^{*}$ acts on $H^{*}(X) \otimes \mathbb{R}^{N}$. However, the action on $H^{*}(X) \otimes \mathbb{R}^{N}$ is clearly independent of the metric, and so the second term of the integrand of (2.21) is independent of $\varepsilon$.

For simplicity, we will write $\left.\frac{d}{d s}\right|_{s=0}$ to mean the derivative at $s=0$ of the analytic continuation of an expression which is defined for $\operatorname{Re} s \gg 0$. Then the rest of (2.21) can be written as the $T$-integral of

$$
\begin{aligned}
& -\left.\frac{d}{d s}\right|_{s=0} \frac{1}{\Gamma(s)} T^{s} \operatorname{Tr}(-)^{F} F \Pi \frac{d \Delta}{d \varepsilon} e^{-T \Delta} \\
& \text { (2.24) }=-\left.\frac{d}{d s}\right|_{s=0} \frac{1}{\Gamma(s)} T^{s} \operatorname{Tr}(-)^{F} F \Pi\{d,[V, \delta]\} e^{-T \Delta} \\
& \text { (2.25) }=-\left.\frac{d}{d s}\right|_{s=0} \frac{1}{\Gamma(s)} T^{s} \operatorname{Tr} \Pi V \\
& \cdot\left[\left(\delta(-)^{F} F d+\delta d(-)^{F} F-(-)^{F} d \delta-d(-)^{F} F \delta\right)\right] e^{-T \Delta} \\
& \text { (2.26) }=-\left.\frac{d}{d s}\right|_{s=0} \frac{1}{\Gamma(s)} T^{s} \operatorname{Tr} \Pi V(-)^{F}(\delta[d, F]+[d, F] \delta) e^{-T \Delta} \\
& \text { (2.27) }=\left.\frac{d}{d s}\right|_{s=0} \frac{1}{\Gamma(s)} T^{s} \operatorname{Tr} \Pi V(-)^{F} \Delta e^{-T \Delta} \\
& \text { (2.28) }=\left.\frac{d}{d s}\right|_{s=0} \frac{1}{\Gamma(s)} T^{s} \operatorname{Tr} \Pi V(-)^{F} \Delta^{\prime} e^{-T \Delta^{\prime}} \\
& \text { (2.29) }=\left.\frac{d}{d s}\right|_{s=0} \frac{1}{\Gamma(s)}\left[-\frac{d}{d T}\left(T^{s} \operatorname{Tr} \Pi V(-)^{F} e^{-T \Delta^{\prime}}\right)\right. \\
& \left.+s T^{s-1} \operatorname{Tr} \Pi V(-)^{F} e^{-T \Delta^{\prime}}\right],
\end{aligned}
$$

so

$$
\frac{d}{d \varepsilon} T_{\rho}=B+C
$$

where

$$
\begin{aligned}
& B=\int_{0}^{\infty}-\left.\frac{d}{d s}\right|_{s=0} \frac{1}{\Gamma(s)} \frac{d}{d T}\left(T^{s} \operatorname{Tr} \Pi V(-)^{F} e^{-T \Delta^{\prime}}\right) d T, \\
& C=\left.\int_{0}^{\infty} \frac{d}{d s}\right|_{s=0} \frac{1}{\Gamma(s)} s T^{s-1} \operatorname{Tr} \Pi V(-)^{F} e^{-T \Delta^{\prime}} d T .
\end{aligned}
$$

Now

$$
B=\left(\lim _{T \rightarrow \infty}-\lim _{T \rightarrow 0}\right)\left(-\left.\frac{d}{d s}\right|_{s=0} \frac{1}{\Gamma(s)}\left(T^{s} \operatorname{Tr} \Pi V(-)^{F} e^{-T \Delta^{\prime}}\right)\right)
$$


Because everything is defined by analytic continuation from $\operatorname{Re} s \gg 0$, both limits vanish and $B=0$.

We now have

$$
\frac{d}{d \varepsilon} T_{\rho}=C=\left.\frac{d}{d s}\right|_{s=0} \frac{1}{\Gamma(s)} s \int_{0}^{\infty} T^{s-1} \operatorname{Tr} \Pi V(-)^{F} e^{-T \Delta^{\prime}} d T .
$$

As $1 / \Gamma(s)$ is $O(s)$, and $\int_{a}^{\infty} T^{s-1} \operatorname{Tr} \Pi V(-)^{F} e^{-T \Delta^{\prime}} d T$ is holomorphic in $s$ for any $a>0$, it follows that

$$
\begin{aligned}
& \frac{d}{d \varepsilon} T_{\rho}=\left.\frac{d}{d s}\right|_{s=0} \frac{1}{\Gamma(s)} s \int_{0}^{a} T^{s-1} \operatorname{Tr} \Pi V(-)^{F} e^{-T \Delta^{\prime}} d T \\
& =\left.\frac{d}{d s}\right|_{s=0} \frac{1}{\Gamma(s)} s\left[\int_{0}^{a} T^{s-1} \operatorname{Tr} \Pi V(-)^{F} e^{-T \Delta} d T\right. \\
& \left.-\left.s^{-1} a^{s} \operatorname{Tr}\right|_{\operatorname{Ker} \Delta} \Pi V(-)^{F}\right] \\
& =\left[\begin{array}{l}
\text { the } T^{0} \text { term in the asymptotic expansion of } \\
\left.\operatorname{Tr} \Pi V(-)^{F} e^{-T \Delta}\right)-\left.\operatorname{Tr}\right|_{\operatorname{Ker} \Delta} \Pi V(-)^{F}
\end{array}\right] .
\end{aligned}
$$

In order to prove the proposition, it is sufficient to show that the asymptotic expansion in $T$ of $\operatorname{Tr} \Pi V(-)^{F} e^{-T \Delta}$ has no $T^{0}$ term, which will be implied if we can show that $\left.\operatorname{Tr} V a(g)^{*} e^{-T \Delta}\right|_{\Lambda^{k}(X)}$ has no $T^{0}$ term for $g \in G$ and $0 \leq k \leq n$.

Let $e^{-T \Delta}{ }_{J I}(g x, x)$ denote the operator kernel for $e^{-T \Delta}$ going from an orthonormal basis $\tau^{I}$ of $\Lambda^{k}(x)$ to an orthonormal basis $\tau^{J}$ of $\Lambda^{k}(g x)$. Let $V_{I J}(x)$ denote the matrix form of the local operator $V$ with respect to this basis. Then we want to look at the asymptotic expansion of

$$
\mathscr{Z} \equiv \int_{X} \sum_{I, J, K} V_{I K}(x)\left\langle\tau_{K}(x),\left(a(g)^{*} \tau_{J}\right)(x)\right\rangle e_{J I}^{-T \Delta}(g x, x) d \operatorname{vol}(x) .
$$

Let us review the results on heat kernel expansions as in, for example, [1], when generalized to the case of the Laplacian acting on differential forms. Let $\eta$ be a bump function on $\mathbb{R}$ with center at the origin and support within $\{r \in \mathbb{R}:|r|<($ the injectivity radius of $X)\}$. Let

$$
S^{(k)}\left(x, x^{\prime}\right)=(4 \pi T)^{-n / 2} e^{-d^{2}\left(x, x^{\prime}\right) /(4 T)} \sum_{l=0}^{k} T^{l} u_{l}\left(x, x^{\prime}\right)
$$

be a parametrix for the heat kernel and put

$$
\begin{gathered}
H^{(k)}\left(x, x^{\prime}\right)=\eta\left(x, x^{\prime}\right) S\left(x, x^{\prime}\right), \\
\mathscr{K}^{(k)}=\left(\partial / \partial_{T}+\Delta\right) H^{(k)} .
\end{gathered}
$$


Let $\left[\mathscr{K}^{(k)}\right]^{* \lambda}$ denote the convolution of $\mathscr{K}^{(k)}$ with itself $\lambda$ times and put

$$
Q^{(k)}=\sum_{\lambda=1}^{\infty}(-1)^{\lambda+1}\left[\mathscr{K}^{(k)}\right]^{* \lambda} \text {. }
$$

Then the heat kernel is

$$
e^{-T \Delta}=H^{(k)}-Q^{(k)} * H^{(k)}
$$

provided $k>n / 2+2$, [1].

Fix $T$ to lie in the interval $(0, a)$ hereafter. Then for $k$ large enough,

$$
R^{(k)}=\lim _{T \rightarrow 0} T^{(n / 2)-k} Q^{(k)}\left(x, x^{\prime}\right)
$$

exists and is bounded in the sup norm on $\left[\Lambda^{p}(X)\right]^{*} \otimes \Lambda^{p}(X)$. Now

$$
\begin{aligned}
\mathscr{Z}=\int_{X} & \sum_{I, J, K} V_{I K}(x)\left\langle\tau_{K}(x),\left(a(g)^{*} \tau_{J}\right)(x)\right\rangle \\
& \times\left[H_{J I}^{(k)}(g x, x)-\sum_{L} \int_{X} Q_{J L}^{(k)}(g x, \tilde{x}) H_{L I}^{(k)}(\tilde{x}, x) d \operatorname{vol}(\tilde{x})\right] d \operatorname{vol}(x) .
\end{aligned}
$$

For small $T, \sum_{L} \int_{X} Q_{J L}^{(k)}(g x, \tilde{x}) H_{L I}^{(k)}(\tilde{x}, x) d \operatorname{vol}(\tilde{x})$ is $O\left(T^{k-n / 2}\right)$. (In fact, it is asymptotic to $T^{k-n / 2} R_{J I}^{(k)}(g x, x)$.) Thus, by choosing $k$ large, we can ensure that the second part of (2.45) does not contribute to the constant term in $T$ of $\mathscr{Z}$, and so we can simply examine the asymptotic expansion of $H$ in order to determine the $T^{0}$ term of $\mathscr{Z}$.

The first part of $\mathscr{Z}$ is

$$
\begin{aligned}
\sum_{l=0} \int_{X} \sum_{I, J, K} V_{I K}(x) \eta(d(x, g x))\left\langle\tau_{K}(x),\left(a(g)^{*} \tau_{J}\right)(x)\right\rangle \\
\quad \times(4 \pi T)^{-n / 2} e^{-d^{2}(x, g x) /(4 T)} \sum_{l=0}^{k} T^{l} u_{l J I}(g x, x) d \operatorname{vol}(x) .
\end{aligned}
$$

Clearly, for any $\varphi>0$, the points $x$ such that $d^{2}(x, g x)>\varphi$ do not contribute to the asyptotic expansion of $\mathscr{Z}$. We know that there is a finite number of components of FIX $(a(g))$ and that these components are boundaryless submanifolds of $X$. It follows that it suffices to integrate the integrand of (2.46) over the union of the tubular neighborhoods of these connected components. Let $\mathscr{C}$ be a connected component of $\operatorname{FIX}(a(g))$ of dimension $c$, and let $\left\{V_{\beta}\right\}$ be a collection of coordinate charts on $\mathscr{C}$ with $\left\{\rho_{\beta}\right\}$ a partition of unity subordinate to $\left\{V_{\beta}\right\}$. By the generalized 
Morse lemma [10], we can choose coordinates $(w, z) \in \mathbb{R}^{c} \oplus \mathbb{R}^{n-c}$ on a tubular neighborhood of $V_{\beta}$ such that $d^{2}(x, g x)=|z|^{2}$. Using the partition of unity, the integral of (2.46) is a sum of terms of the form

$$
T^{l-n / 2} \int_{\text {TUB NBHD }} q(w, z) e^{-|z|^{2} / 4 T} \rho_{\beta}(w),
$$

with $q$ being a $C^{\infty}$ function on the tubular neighborhood. (Note that it is irrelevant whether $\mathscr{C}$ is orientable.) We can extend $q$ to a $C_{0}^{\infty}$ function $\widetilde{q}$ on $\mathbb{R}^{N}$ and thereby integrate over $z \in \mathbb{R}^{n-c}$ without changing the asymptotics of (2.47) in $T$. Thus we have an integral of the form

$$
T^{l-n / 2} \int \widetilde{q}(w, z) e^{-|z|^{2} / 4 T} \rho_{\beta}(w) d^{c} w d^{n-c} z .
$$

Doing the $w$-integral gives something of the form

$$
\begin{aligned}
& T^{l-n / 2} \int r(z) e^{-|z|^{2} / 4 T} d^{n-c} z \\
& \quad=T^{(n-c) / 2} T^{l-n / 2} \int r\left(z T^{1 / 2}\right) e^{-|z|^{2} / 4} d^{n-c} z,
\end{aligned}
$$

with $r \in C_{0}^{\infty}\left(\mathbb{R}^{n-c}\right)$. Expanding $r$ in a Taylor's series gives an expansion of the last integral in integer powers of $T$, with error of arbitrarily high order in $T$. Thus, all of the powers of $T$ in the asymptotic expansion of $\mathscr{Z}$ lie in $T^{-c / 2+\mathbb{Z}}$. Because $a(g)$ is an orientation-preserving isometry, $\mathscr{C}$ must be of even codimension, $c$ must be odd, and so there is no $T^{0}$ term in the asymptotic expansion of $\mathscr{Z}$.

\section{Product formulas}

Let $c$ denote the character of the representation $\rho$, i.e., $c(g)=\operatorname{Tr} \rho(g)$. Note that we can write $T_{\rho}$ as

$$
T_{\rho}=\frac{1}{|G|} \sum_{g} c(g) t(g)
$$

where

$$
t(g)=\left.\frac{d}{d s}\right|_{s=0} \frac{1}{\Gamma(s)} \int_{0}^{\infty} T^{s-1} \operatorname{Tr} a\left(g^{-1}\right)^{*}(-)^{F} F e^{-T \Delta^{\prime}} d T .
$$

Let $L(g)$ denote the Lefschetz number of $g$.

Proposition 3. Suppose that $X_{1}$ and $X_{2}$ are closed oriented Riemannian manifolds. Let $X_{1} \times X_{2}$ have the product metric. If $G_{1}$ acts on $X_{1}$ by 
isometries, and $G_{2}$ acts on $X_{2}$ by isometries such that the product action on $X_{1} \times X_{2}$ is orientation-preserving, then for $g_{1} \in G_{1}$ and $g_{2} \in G_{2}$,

$$
t\left(g_{1}, g_{2}\right)=t\left(g_{1}\right) L\left(g_{2}\right)+L\left(g_{1}\right) t\left(g_{2}\right) \text {. }
$$

Proof. Consider the operators $\Delta=\Delta_{1} \otimes I+I \otimes \Delta_{2}$ and $F=F_{1} \otimes I+$ $I \otimes F_{2}$, which we will abbreviate by $\Delta_{1}+\Delta_{2}$ and $F_{1}+F_{2}$ respectively, acting on

$$
\Lambda^{*}\left(X_{1} \times X_{2}\right)=\Lambda^{*}\left(X_{1}\right) \otimes \Lambda^{*}\left(X_{2}\right)
$$

Then

$$
\begin{aligned}
t\left(g_{1}, g_{2}\right)=\left.\frac{d}{d s}\right|_{s=0} \frac{1}{\Gamma(s)} \int_{0}^{\infty} T^{s-1} & \operatorname{Tr} a\left(g_{1}^{-1}, 1\right)^{*} a\left(1, g_{2}^{-1}\right)^{*} \\
& \times(-)^{F_{1}+F_{2}}\left(F_{1}+F_{2}\right) e^{-T\left(\Delta_{1}+\Delta_{2}\right)^{\prime}} d T .
\end{aligned}
$$

Because the eigenvectors of $\Delta_{1}+\Delta_{2}$ with nonzero eigenvalue are of the form $v_{1} \otimes v_{2}$, where $v_{1}$ is an eigenvector of $\Delta_{1}$, and $v_{2}$ is an eigenvector of $\Delta_{2}$ such that at least one of $v_{1}$ and $v_{2}$ has a nonzero eigenvalue, it follows that (3.4) equals

$$
\begin{aligned}
&\left.\frac{d}{d s}\right|_{s=0} \frac{1}{\Gamma(s)} \int_{0}^{\infty} T^{s-1} \operatorname{Tr} a\left(g_{1}^{-1}\right)^{*}(-)^{F_{1}} F_{1} e^{-T \Delta_{1}^{\prime}} \operatorname{Tr} a\left(g_{2}^{-1}\right)^{*}(-)^{F_{2}} e^{-T \Delta_{2}} d T \\
&+\left.\frac{d}{d s}\right|_{s=0} \frac{1}{\Gamma(s)} \int_{0}^{\infty} T^{s-1} \operatorname{Tr} a\left(g_{2}^{-1}\right)^{*}(-)^{F_{2}} F_{2} e^{-T \Delta_{2}^{\prime}} \operatorname{Tr} a\left(g_{1}^{-1}\right)^{*} \\
& \times(-)^{F_{1}} e^{-T \Delta_{1}} d T .
\end{aligned}
$$

We have

$$
L\left(g_{2}\right)=\operatorname{Tr} a\left(g_{2}^{-1}\right)^{*}(-)^{F_{2}} e^{-T \Delta_{2}},
$$

(see, for example, [9]) and similarly for $L\left(g_{1}\right)$. Hence the proposition follows. q.e.d.

Let $\chi_{\rho}$ generally denote the Euler character of the $d$-complex of $\rho$ equivariant $\mathbb{R}^{N}$-valued differential forms.

Proposition 4. Suppose that $\rho_{1}$ is an orthogonal representation of $G_{1}$, and $\rho_{2}$ is an orthogonal representation of $G_{2}$. With the hypotheses of Proposition 3,

$$
T_{\rho_{1} \otimes \rho_{2}}\left(X_{1} \times X_{2}\right)=T_{\rho_{1}}\left(X_{1}\right) \chi_{\rho_{2}}\left(X_{2}\right)+T_{\rho_{2}}\left(X_{2}\right) \chi_{\rho_{1}}\left(X_{1}\right) .
$$


Proof.

$$
\begin{aligned}
T_{\rho_{1} \otimes \rho_{2}}= & \frac{1}{\left|G_{1}\right|\left|G_{2}\right|} \sum_{g_{1}, g_{2}} c_{1}\left(g_{1}\right) c_{2}\left(g_{2}\right) t\left(g_{1}, g_{2}\right) \\
= & \frac{1}{\left|G_{1}\right|\left|G_{2}\right|} \sum_{g_{1}, g_{2}} c_{1}\left(g_{1}\right) c_{2}\left(g_{2}\right)\left(t\left(g_{1}\right) L\left(g_{2}\right)+L\left(g_{1}\right) t\left(g_{2}\right)\right) \\
= & \frac{1}{\left|G_{1}\right|} \sum_{g_{1}} c_{1}\left(g_{1}\right) t\left(g_{1}\right) \frac{1}{\left|G_{2}\right|} \sum_{g_{2}} c_{2}\left(g_{2}\right) L\left(g_{2}\right) \\
& +\frac{1}{\left|G_{2}\right|} \sum_{g_{2}} c_{2}\left(g_{2}\right) t\left(g_{2}\right) \frac{1}{\left|G_{1}\right|} \sum_{g_{1}} c_{1}\left(g_{1}\right) L\left(g_{1}\right) \\
= & T_{\rho_{1}} \chi_{\rho_{2}}+T_{\rho_{2}} \chi_{\rho_{1}} \text {. q.e.d. }
\end{aligned}
$$

Given an action of a finite group $G$ on $X$, let $\rho_{H}$ denote the (virtual) representation of $G$ given by the difference of the $G$-module $H^{\text {even }}(X)$ and the $G$-module $H^{\text {odd }}(X)$. Because $G$ preserves the inner product on $H^{*}(X)$ induced from the de Rham isomorphism, $\rho_{H}$ is an orthogonal (virtual) representation. We can derive for virtual representations $\rho, t \rho$, by additivity.

Proposition 5. If $G$ acts on $X_{1}$ and on $X_{2}$, then for the diagonal action of $G$ on $X_{1} \times X_{2}$,

$$
T_{\rho}\left(X_{1} \times X_{2}\right)=T_{\rho \otimes \rho_{H_{2}}}\left(X_{1}\right)+T_{\rho \otimes \rho_{H_{1}}}\left(X_{2}\right) .
$$

Proof.

$$
\begin{aligned}
T_{\rho} & =\frac{1}{|G|} \sum_{g} c(g) t(g, g) \\
& =\frac{1}{|G|} \sum_{g} c(g)\left(t_{1}(g) L_{2}(g)+L_{1}(g) t_{2}(g)\right) \\
& =\frac{1}{|G|} \sum_{g} c(g) L_{2}(g) t_{1}(g)+\frac{1}{|G|} \sum_{g} c(g) L_{1}(g) t_{2}(g) \\
& =T_{\rho \otimes \rho_{H_{2}}}\left(X_{1}\right)+T_{\rho \otimes \rho_{H_{1}}}\left(X_{2}\right) .
\end{aligned}
$$

Proposition 6. Suppose that $G$ acts by orientation-preserving diffeomorphisms on $X, H$ is a subgroup of $G, \rho$ is an orthogonal representation of $H$, and $\widetilde{\rho}$ is the induced representation of $G$. Then $T_{\widetilde{\rho}}=T_{\rho}$.

Proof. Note that if the character of $\rho$ is given by $c(h)$, then the character of $\tilde{\rho}$ is given by

$$
\widetilde{c}(g)=\frac{1}{|H|} \sum_{g^{\prime}, h: g^{\prime} h\left(g^{\prime}\right)^{-1}=g} c(h) .
$$


Then

$$
T_{\widetilde{\rho}}=\frac{1}{|G|} \frac{1}{|H|} \sum_{g^{\prime} h: g^{\prime} h\left(g^{\prime}\right)^{-1}=g} c(h) t(g) .
$$

Now

$$
\begin{aligned}
t\left(g^{\prime} h\left(g^{\prime}\right)^{-1}\right)= & \left.\frac{d}{d s}\right|_{s=0} \frac{1}{\Gamma(s)} \int_{0}^{\infty} T^{s-1} \operatorname{Tr} a\left(g^{\prime-1}\right)^{*} a\left(h^{-1}\right)^{*} \\
& \cdot a\left(g^{\prime}\right)^{*}(-)^{F} F e^{-T \Delta^{\prime}} d T \\
= & \left.\frac{d}{d s}\right|_{s=0} \frac{1}{\Gamma(s)} \int_{0}^{\infty} T^{s-1} \operatorname{Tr} a\left(g^{\prime-1}\right)^{*} a\left(h^{-1}\right)^{*} \\
& \cdot(-)^{F} F e^{-T \Delta^{\prime}} a\left(g^{\prime}\right)^{*} d T \\
= & t(h) .
\end{aligned}
$$

Thus

$$
\begin{aligned}
T_{\widetilde{\rho}} & =\frac{1}{|G|} \frac{1}{|H|} \sum_{g^{\prime}, h} c(h) t(h) \\
& =\frac{1}{|H|} \sum_{h} c(h) t(h) \\
& =T_{\rho} .
\end{aligned}
$$

\section{Computations of $T$ for orthogonal actions on spheres}

We now compute the analytic torsion for certain group actions on a sphere. Suppose that $\sigma: G \rightarrow \mathrm{SO}(2 n)$ is a representation of a finite group into the special orthogonal group, and consider the induced action on $S^{2 n-1}$. Let $\rho: G \rightarrow \mathrm{O}(N)$ be an orthogonal representation of $G$ such that $\sum_{g} c(g)=0$, where $c$ denotes the character of the representation $\rho$, i.e., $\rho$ has no trivial component. By equations (10) and (11) of Ray [16], we have

$$
T_{\rho}=\frac{1}{|G|} \sum_{j=1}^{|G|} \log \left|e^{2 \pi i j /|G|}-1\right|\left(\sum_{g} c(g) \operatorname{Tr} \sigma\left(g^{j}\right)\right) .
$$

Note that the $g$ summation must be done first in this equation in order to get a well-defined answer.

For future use, let us compute the analytic torsion for a cyclic subgroup of $G$. Let $g$ be a fixed element of $G$, and let us compute the contribution 
to $T_{\rho}$ coming from the subgroup generated by $g$. Choose $k$ such that $|G|+k$, and consider

$$
F_{k} \equiv \frac{1}{|G|} \sum_{j=1}^{|G|} \log \left|e^{2 \pi i j /|G|}-1\right| \sum_{r=1}^{|G|} e^{-2 \pi i r k /|G|} \operatorname{Tr} \sigma\left(g^{j r}\right) .
$$

Suppose that the normal form for $\sigma(g)$ has block matrices

$$
\left(\begin{array}{cc}
\cos 2 \pi \nu_{l} /|G| & \sin 2 \pi \nu_{l} /|G| \\
-\sin 2 \pi \nu_{l} /|G| & \cos 2 \pi \nu_{l} /|G|
\end{array}\right)_{l=1}^{n}
$$

along the diagonal. If $\nu_{l}$ is nonzero, define $\alpha(l)$ by

$$
\alpha(l) \frac{\nu_{l}}{\operatorname{gcd}\left(\nu_{l},|G|\right)} \equiv 1 \quad \bmod \frac{|G|}{\operatorname{gcd}\left(\nu_{l},|G|\right)} .
$$

Let us write $\operatorname{gcd}(l)$ hereafter for $\operatorname{gcd}\left(\nu_{l},|G|\right)$.

\section{Proposition 7.}

$$
F_{k}=\sum_{\substack{l=1 \\ \operatorname{gcd}(l) \mid k}}^{|G|} \log \left|1-e^{2 \pi i k \alpha(l) /|G|}\right|
$$

Proof. We have

$$
\begin{array}{r}
F_{k}=\frac{1}{|G|} \sum_{j=1}^{|G|} \log \left|e^{2 \pi i j /|G|}-1\right| \sum_{r=1}^{|G|} e^{-2 \pi i r k /|G|} \\
\times \sum_{l=1}^{n}\left(e^{2 \pi i j r \nu_{l} /|G|}+e^{-2 \pi i j r \nu_{l} /|G|}\right) \\
=\sum_{j=1}^{|G|} \log \left|e^{2 \pi i j /|G|}-1\right| \sum_{\substack{l=1 \\
j \nu_{l} \equiv \pm k \bmod |G|}}^{n} 1 .
\end{array}
$$

Now $j \nu_{l} \equiv \pm k \bmod |G|$ has a solution if and only if $\operatorname{gcd}(l) \mid k$, in which case the solutions for $j$ are

$$
j \in\left\{ \pm \frac{k \alpha(l)}{\operatorname{gcd}(l)}+s \frac{|G|}{\operatorname{gcd}(l)}\right\}_{s=0}^{\operatorname{gcd}(l)-1} .
$$


This gives

$$
\begin{aligned}
F_{k} & =\sum_{l=1}^{n} \sum_{\substack{j=1 \\
j \nu l} \pm k \bmod |G|}^{|G|} \log \left|e^{2 \pi i j /|G|}-1\right| \\
& =\sum_{\substack{l=1 \\
\operatorname{gcd}(l) \mid k}}^{n} \sum_{s=0}^{\operatorname{gcd}(l)-1} \log e^{2 \pi i(s|G| \pm k \alpha(l)) /(|G| \operatorname{gcd}(l))}-1 \mid \\
& =\sum_{\substack{l=1 \\
\operatorname{gcd}(l) \mid k}}^{n} \sum_{s=0}^{\operatorname{gcd}(l)-1} \log \left|e^{2 \pi i s / \operatorname{gcd}(l)}-e^{\mp 2 \pi i k \alpha(l) /(|G| \operatorname{gcd}(l))}\right| \\
& =\sum_{\substack{l=1 \\
\operatorname{gcd}(l) \mid k}}^{n} \log \left|1-e^{\mp 2 \pi i k \alpha(l) /|G|}\right| \\
& =\sum_{\substack{l=1 \\
\operatorname{gcd}(l) \mid k}}^{n} \log \left|1-e^{2 \pi i k \alpha(l) /|G|}\right| .
\end{aligned}
$$

\section{The combinatorial torsion for finite group actions}

An expression for the Reidemeister torsion for actions of finite groups on PL $G$-manifolds was developed by Rothenberg [19]. To make this paper self-contained, we will summarize a simplified version of this work which is sufficient for our purposes. This approach is based on the work of Milnor in [14]. The reader may wish to refer to this reference for information on the torsion of a complex.

Let $G$ be a finite group, and let $K$ be a finite regular simplicial $G$ complex [2]. Let $\rho: G \rightarrow O(N)$ be an orthogonal representation of $G$. Consider the equivariant cochain groups

$$
C^{k}(K, \rho)=\left\{\omega \in C^{k}(K) \otimes \mathbb{R}^{N}: \text { for all } g \in G, \rho(g) a\left(g^{-1}\right)^{*} \omega=\omega\right\},
$$

where $a\left(g^{-1}\right)^{*}$ denotes the action of $g \in G$ on $C^{k}(K)$, and the complex

$$
C^{0}(K, \rho) \stackrel{d_{c}}{\longrightarrow} C^{1}(K, \rho) \stackrel{d_{c}}{\longrightarrow} \cdots \stackrel{d_{c}}{\longrightarrow} C^{n}(K, \rho) .
$$

In order to define the torsion of the complex [14] we must give volume forms for the cohomology groups $H^{k}(K, \rho)$ of the complex and for the cochain groups $C^{k}(K, \rho)$. As in $\S I I$, when $K$ is a triangulation of a 
smooth compact oriented $n$-dimensional $G$-manifold $X$, the de Rham isomorphism between $\operatorname{Ker} \Delta_{k}$ and $H^{k}(K, \rho)$ gives the latter the inner product structure induced from $\mathscr{\ell}^{k}(X) \otimes \mathbb{R}^{N}$.

Let us define an inner product on $C^{k}(K, \rho)$. If we take the $k$-simplices to form a preferred basis of $C^{k}(K)$, and we use the standard inner product on $\mathbb{R}^{N}$, we obtain an inner product on $C^{k}(K) \otimes \mathbb{R}^{N}$. Give $C^{k}(K, \rho)$ the inner product that is induced as a subspace of $C^{k}(K) \otimes \mathbb{R}^{N}$. The volume form on $C^{k}(K, \rho)$ is that determined by this inner product.

Proposition 8. The R-torsion $\tau$ of the complex (5.2) is invariant under equivariant subdivision of $K$.

Proof. As the torsion of the cochain complex is the same (up to a sign) as that of the chain complex, it suffices to prove the proposition for the chain complex

$$
C_{n}(K, \rho) \stackrel{\partial}{\longrightarrow} C_{n-1}(K, \rho) \stackrel{\partial}{\longrightarrow} \cdots \stackrel{\partial}{\longrightarrow} C_{0}(K, \rho),
$$

where $C_{k}(K, \rho)$ denotes the $\rho$-equivariant elements of $C_{k}(K) \otimes \mathbb{R}^{N}$. The proof is now a straightforward adaptation of the invariance proof in $\S 7$ of [14]. We omit the details. If $L$ is a subcomplex of $K$, then the same construction goes through for $K$ rel $L$. q.e.d.

One can define a more general combinatorial torsion using the inner product

$$
\left\langle\eta_{1}, \eta_{2}\right\rangle=\sum_{\sigma} w\left(H_{\sigma}\right)\left\langle\eta_{1}(\sigma), \eta_{2}(\sigma)\right\rangle_{\mathbb{R}^{N}},
$$

where $\eta_{1}$ and $\eta_{2}$ are elements of $C^{k}(K, \rho)$, the sum is over $k$-simplices $\sigma, H_{\sigma}$ is the isotropy group of $\sigma$, and $w$ is a fixed positive function from the set of conjugacy classes of subgroups of $G$ to $\mathbb{R}$. The torsion so defined is again subdivision invariant. We consider the special case of $w=1$ hereafter in order to ensure that the product formula holds for the combinatorial torsion. This corrects the choice of $w$ given in [12].

\section{Computation of $\tau$ for orthogonal actions on spheres}

Let us first consider the case of cyclic actions on $S^{1}$.

Proposition 9. Suppose that $\mathbb{Z}_{p}$ acts on $S^{1}$ by

$$
\left.a(r) e^{i \theta}\right)=e^{i(\theta+2 \pi r \nu / p)},
$$

where $0 \leq r<p$ and $0 \leq \nu<p$. Let $\operatorname{gcd}$ denote $\operatorname{gcd}(\nu, p)$ and consider 
the character $c(r)$ of the two-dimensional representation of $\mathbb{Z}_{p}$ given by

$$
c(r)=2 \cos (2 \pi k r / p) \text {. }
$$

Suppose that $\mathrm{gcd} \mid k$ (in order to have a nontrivial equivariant cochain complex) and that $p+k$ (in order to have an acyclic equivariant cochain complex). Let $\alpha$ satisfy

$$
\alpha \frac{\nu}{\mathrm{gcd}} \equiv 1 \quad \bmod \frac{p}{\mathrm{gcd}}
$$

Then the torsion of the cochain complex is given by

$$
\tau(p, k)=\log |1-\exp (2 \pi i k \alpha / p)| .
$$

Proof. Let $K$ be the triangulation of $S^{1}$ with $p$ vertices and $p$ edges. The equivariant cochain complex of $K$ is the same as that of the free action of $\mathbb{Z}_{p / \mathrm{gcd}}$ on $K$ with action

$$
a(t)\left(e^{i \theta}\right)=e^{i(\theta+2 \pi t(\nu / \mathrm{gcd}) /(p / \mathrm{gcd}))}, \quad 0 \leq t<p / \mathrm{gcd},
$$

and character

$$
c(t)=2 \cos (2 \pi t(k / \mathrm{gcd}) /(p / \mathrm{gcd})) .
$$

However, the torsion of this complex is the same as the standard Reidemeister torsion of $S^{1}$ using a flat $\mathbb{R}^{2}$ bundle whose holonomy around $S^{1}$ is given by

$$
\left(\begin{array}{cc}
\cos (2 \pi \alpha(k / \text { gcd }) /(p / \text { gcd })) & \sin (2 \pi \alpha(k / \text { gcd }) /(p / g c d)) \\
-\sin (2 \pi \alpha(k / \text { gcd }) /(p / \text { gcd })) & \cos (2 \pi \alpha(k / \text { gcd }) /(p / \text { gcd }))
\end{array}\right) .
$$

This is easily computed to be

$$
\log \left|1-e^{2 \pi i \alpha(k / \mathrm{gcd}) /(p / \mathrm{gcd})}\right|=\log |1-\exp (2 \pi \mathrm{ik} \alpha / \mathrm{p})| .
$$

(We have used a normalization on $\tau$ to agree with the normalization of $\S I V$.$) q.e.d.$

We will now compute $\tau$ for a cyclic group of special orthogonal actions on a sphere.

Proposition 10. Let $\sigma: \mathbb{Z}_{p} \rightarrow \mathrm{SO}(2 n)$ be a special orthogonal representation of the cyclic group $\mathbb{Z}_{p}$, and consider the induced action on $S^{2 n-1}$. Suppose that the normal form of $\sigma(1)$ has the block matrices

$$
\left(\begin{array}{cc}
\cos 2 \pi \nu_{l} / p & \sin 2 \pi \nu_{l} / p \\
-\sin 2 \pi \nu_{l} / p & \cos 2 \pi \nu_{l} / p
\end{array}\right)_{l=1}^{n}
$$

along the diagonal. Consider the character $c(r)$ of the two-dimensional representation of $\mathbb{Z}_{p}$ given by

$$
c(r)=2 \cos (2 \pi k r / p), \quad 0 \leq r<p,
$$


with $p+k$ (in order to have an acyclic complex). Let $\operatorname{gcd}(l)$ denote $\operatorname{gcd}\left(\nu_{l}, p\right)$, and for nonzero $\nu_{l}$, define $\alpha(l)$ by

$$
\alpha(l) \frac{\nu_{l}}{\operatorname{gcd}\left(\nu_{l}, p\right)} \equiv 1 \quad \bmod \frac{p}{\operatorname{gcd}\left(\nu_{l}, p\right)} .
$$

Then the torsion is given by

$$
\tau=\sum_{\substack{l \\ \operatorname{gcd}(l) \mid k}} \log \left|1-e^{2 \pi i k \alpha(l) / p}\right| .
$$

Proof. The key of the proof is to consider the properties of $\tau$ under the join operation [19]. If $X$ and $Y$ are $G$-simplicial complexes, then there is a relative isomorphism between $(X \# Y, X \cup Y)$ and $\left(S(X \times Y), p_{0} \cup p_{1}\right)$. If $\mathscr{H}$ denotes the homology sequence of the pair $(X \# Y, X \cup Y)$, then by Theorem 3.2 of [14], we have that

$$
\begin{aligned}
\tau(X \# Y) & =\tau(X)+\tau(Y)+\tau(X \# Y, X \cup Y)+\tau(\mathscr{H}) \\
& =\tau(X)+\tau(Y)+\tau\left(S(X \times Y), p_{0} \cup p_{1}\right)+\tau(\mathscr{H}) \\
& =\tau(X)+\tau(Y)-\tau(X \times Y)+\tau(\mathscr{H}) .
\end{aligned}
$$

If the representation is such that $H^{*}(X \# Y, \rho), H^{*}(X, \rho)$, and $H^{*}(Y, \rho)$ vanish, it follows that $\tau(\mathscr{H})=0$, and so

$$
\tau(X \# Y)=\tau(X)+\tau(Y)-\tau(X \times Y) .
$$

In our case we can construct the $\mathbb{Z}_{p}$ action on $S^{2 n-1}$ as a repeated join of actions on $S^{1}$. As the Lefschetz number of an orientation-preserving action on an odd-dimensional sphere vanishes, it follows from Propositions 3 and $5^{\prime}$ (at the end of this section) that with each join,

$$
\tau(X \# Y)=\tau(X)+\tau(Y) .
$$

Using the result of Proposition 9, the proposition follows. q.e.d.

We now show that $T_{\rho}$ and $\tau_{\rho}$ coincide for all special orthogonal actions on odd-dimensional spheres. The first step is to show that $\tau_{\rho}$ can be written linearly in terms of the character $c$ of the representation $\rho$, as in (3.1).

Proposition 11. With the hypotheses of $\S \mathrm{V}, \tau_{\rho}$ can be written in the form

$$
\tau=\frac{1}{|G|} \sum_{g} c(g) t^{\prime}(g)
$$

for some function $t^{\prime}$ on $G$. 
Proof. Let us define the adjoint $\delta_{c}$ to the $d_{c}$ operator using the inner product on $C^{*}(K, \rho)$ defined in $\S \mathrm{V}$. Define the combinatorial Laplacian $\Delta_{c}$ by

$$
\Delta_{c}=d_{c} \delta_{c}+\delta_{c} d_{c}
$$

We would like to write the torsion $\tau$ as

$$
\left.\frac{d}{d s}\right|_{s=0} \frac{1}{\Gamma(s)} \int_{0}^{\infty} T^{s-1} \operatorname{Tr}(-)^{F} F e^{-T} \Delta_{c}^{\prime} d T,
$$

where the trace is over endomorphisms of $C^{*}(K, \rho)$, but this is not quite true. The expression (6.18) would give the torsion for the complex (5.2) if we were to use the volume form $\nu_{i}$ on $H^{i}(K, \rho)$ that is induced from the inner product on $C^{i}(K, \rho)$, but we instead want to use the volume form $\mu_{i}$ on $H^{i}(K, \rho)$ that is induced from the inner product on $\mathscr{H}^{i}(X, \rho)$. In order to correct for this, we must add a factor

$$
\sum_{i}(-1)^{i} \ln \left(\mu_{i} / \nu_{i}\right)
$$

to (6.18). Let $A: \mathscr{H}^{q}(X, \rho) \rightarrow C^{q}(K, \rho)$ be the de Rham operator, and let $A^{*}$ be its dual with respect to the mentioned inner products. Then the correction factor can be written as

$$
-\left.\operatorname{Tr}(-)^{F} \ln \left(A A^{*}\right)\right|_{H^{*}(K, \rho)},
$$

or, equivalently,

$$
-\left.\lim _{T \rightarrow \infty} \operatorname{Tr}(-)^{F} \ln \left(A A^{*}+\Delta_{c}\right) e^{-T} \Delta_{C}\right|_{C^{*}(K, \rho)} .
$$

Thus we have

$$
\begin{aligned}
\tau= & \left.\frac{d}{d s}\right|_{s=0} \frac{1}{\Gamma(s)} \int_{0}^{\infty} T^{s-1} \operatorname{Tr}(-)^{F} F e^{-T} \Delta_{c}^{\prime} d T \\
& -\lim _{T \rightarrow \infty} \operatorname{Tr}(-)^{F} \ln \left(A A^{*}+\Delta_{c}\right) e^{-T} \Delta_{c},
\end{aligned}
$$

where the traces are over endomorphisms of $C^{*}(K, \rho)$. Note that we have a projection operator $\Pi_{c}$ from $C^{*}(K) \otimes \mathbb{R}^{N}$ to $C^{*}(K, \rho)$ given by

$$
\Pi_{c}=\frac{1}{|G|} \sum_{g \in G} \rho(g) a\left(g^{-1}\right)^{*} \text {. }
$$

Then we can write

$$
\begin{aligned}
\tau & =\left.\frac{1}{|G|} \frac{d}{d s}\right|_{s=0} \sum_{g} \frac{1}{\Gamma(s)} \int_{0}^{\infty} T^{s-1} \operatorname{Tr}(-)^{F} F \rho(g) a\left(g^{-1}\right)^{*} e^{-T} \Delta_{c}^{\prime} d T \\
& -\frac{1}{|G|} \sum_{g} \lim _{T \rightarrow \infty} \operatorname{Tr}(-)^{F} \rho(g) a\left(g^{-1}\right)^{*} \ln \left(A A^{*}+\Delta_{c}\right) e^{-T} \Delta_{c}^{\prime}
\end{aligned}
$$


where the traces are now over endomorphisms of $C^{*}(K) \otimes \mathbb{R}^{N}$. Equivalently, we can write

$$
\tau=\frac{1}{|G|} \sum_{g} c(g) t^{\prime}(g)
$$

where

$$
\begin{aligned}
t^{\prime}(g)= & \left.\frac{d}{d s}\right|_{s=0} \frac{1}{\Gamma(s)} \int_{0}^{\infty} T^{s-1} \operatorname{Tr}(-)^{F} F a\left(g^{-1}\right)^{*} e^{-T} \Delta_{c}^{\prime} d T \\
& -\lim _{T \rightarrow \infty} \operatorname{Tr}(-)^{F} a\left(g^{-1}\right)^{*} \ln \left(A A^{*}+\Delta_{c}\right) e^{-T} \Delta_{c}^{\prime},
\end{aligned}
$$

and the traces are now over endomorphisms of $C^{*}(K)$.

Proposition 12. Let $\sigma: G \rightarrow \mathrm{SO}(2 n)$ be a special orthogonal representation of a finite group $G$, and consider the induced action on $S^{2 n-1}$. Let $\rho: G \rightarrow O(N)$ be an orthogonal representation of $G$. Then $T_{\rho}=\tau_{\rho}$.

Proof. Let $\langle g\rangle$ denote the subgroup of $G$ generated by an element $g$ of order $p$. Recall that $t$ is a function on $G$ such that (3.1) holds. By Propositions 7 and 10, we have that

$$
\sum_{r=1}^{p} e^{-2 \pi i r k / p}\left(t-t^{\prime}\right)\left(g^{r}\right)=0,
$$

whenever $p+k$. That is, the discrete Fourier transform of the function $r \rightarrow\left(t-t^{\prime}\right)\left(g^{r}\right)$ has support at $k=0$. Thus $\left(t-t^{\prime}\right)\left(g^{r}\right)$ is independent of $r$, and equals $\left(t-t^{\prime}\right)(e)$. Therefore $\left(t-t^{\prime}\right)(g)=\left(t-t^{\prime}\right)(e)$ for all $g \in G$. Now $\left(t-t^{\prime}\right)(e)$ is the difference between the ordinary analytic torsion and the Reidemeister torsion for the space $S^{2 n-1}$. By the result of [4], [15], we know that this vanishes. q.e.d.

Let us note that the following analogues of Propositions 2-6 hold for $\tau_{\rho}[19]:$

Proposition 2'. Let $g(\varepsilon)$ be a smooth 1-parameter family of G-invariant metrics on $X$. Then

$$
\frac{d}{d \varepsilon} \tau_{\rho}=\left.\operatorname{Tr}\right|_{\operatorname{Ker} \Delta_{c}} \Pi(-)^{F}\left(\frac{d}{d \varepsilon} *\right) *^{-1} .
$$

Proposition 3'. Suppose that $X_{1}$ and $X_{2}$ are closed oriented manifolds. If $G_{1}$ acts on $X_{1}$ and $G_{2}$ acts on $X_{2}$ such that the product action of $G_{1} \times G_{2}$ on $X_{1} \times X_{2}$ is orientation-preserving, then for $g_{1} \in G_{1}$ and $g_{2} \in G_{2}$,

$$
t^{\prime}\left(g_{1}, g_{2}\right)=t^{\prime}\left(g_{1}\right) L\left(g_{2}\right)+L\left(g_{1}\right) t^{\prime}\left(g_{2}\right) \text {. }
$$

Proposition 4'. Suppose that $\rho_{1}$ is an orthogonal representation of $G_{1}$ and $\rho_{2}$ is an orthogonal representation of $G_{2}$. With the hypotheses of 
Proposition 3,

$$
\tau_{\rho_{1} \otimes \rho_{2}}\left(X_{1} \times X_{2}\right)=\tau_{\rho_{1}}\left(X_{1}\right) \chi_{\rho_{2}}\left(X_{2}\right)+\tau_{\rho_{2}}\left(X_{2}\right) \chi_{\rho_{1}}\left(X_{1}\right) .
$$

Proposition $5^{\prime}$. If $G$ acts on $X_{1}$ and $X_{2}$, then for the diagonal action of $G$ on $X_{1} \times X_{2}$,

$$
\tau_{\rho}\left(X_{1} \times X_{2}\right)=\tau_{\rho \otimes \rho_{H_{1}}}\left(X_{1}\right)+\tau_{\rho \otimes \rho_{H_{2}}}\left(X_{2}\right) .
$$

Proposition 6'. Suppose that $G$ acts by orientation-preserving diffeomorphisms on $X, H$ is a subgroup of $G, \rho$ is an orthogonal representation of $H$, and $\widetilde{\rho}$ is the induced representation of $G$. Then $\tau_{\rho}=\tau_{\tilde{\rho}}$.

Note. We have shown that $\tau$ and $T$ coincide for all special orthogonal group actions on odd-dimensional spheres. They definitely do not coincide for special orthogonal actions on even-dimensional spheres. By Proposition 1, $T$ always vanishes in the even-dimensional case. However, $\tau$ does not vanish even for cyclic actions. This can be seen by building a cyclic action using joins as in Proposition 10. We will discuss the lack of equality further in $\S$ VIII.

\section{Equality of $T$ and $\tau$ for odd-dimensional manifolds}

We now show that $T$ equals $\tau$ for orientation-preserving finite group actions on odd-dimensional closed oriented manifolds by following the method of Müller [15]. This consists of proving the equality for special orthogonal actions on spheres and using cobordism methods to pass to the general case. First let us review equivariant surgery.

Let $X$ be as in $\S I I$ and let $f$ be a $G$-Morse function (this exists by [20]). As $G$ is finite, $f$ is simply an ordinary Morse function which happens to be $G$-invariant, and so has a finite number of critical points. Let $\mathscr{B}$ be an orbit of critical points of index $i$, and let $V$ and $W$ denote $G$-vector bundles over $\mathscr{B}$ of dimensions $n-i$ and $i$ respectively. Let $V(1)$ and $W(1)$ denote the unit disk bundles of $V$ and $W$, and call $V(1) \oplus W(1)$ a handle-bundle of index $i$. If $c$ is a critical value of $f$, then for small $\varepsilon, f^{-1}((-\infty, c+\varepsilon))$ differs from $f^{-1}((-\infty, c-\varepsilon))$ by the addition of a finite number of handle-bundles in a way analogous to the case of ordinary Morse theory [20].

In order to obtain a $G$-cobordism between $X \cup(-X)$ and a disjoint union of spheres, let us consider the space $X \times I$ and the function $F: X \times$ $I \rightarrow \mathbb{R}$ given by $F(x, t)=4 t(1-t)(1+f(x))$. As in [4], decomposing $X \times I$ via the handle-bundle additions specified by $F$ corresponds to 
building $X \cup(-X)$ from $S^{n}$ 's by an iterative process, each step of which consists of removing a region of the form $\partial V(1) \oplus W(1)$ and attaching $V(1) \oplus \partial W(1)$. The only point to check is that the group acts orthogonally on the $S^{n}$ 's in the decomposition. The $S^{n}$ 's are in 1-1 correspondence with the cricial points of $F$ of index $n+1$, which are the points $\left(1 / 2, x_{i}\right)$, where $x_{i} \in X$ is a critical point for $f$ of index $n$. The sphere $S^{n}$ is given as the component of $F^{-1}\left(1+f\left(x_{i}\right)-\varepsilon\right)$, for $\varepsilon$ small, which is located near $\left(1 / 2, x_{i}\right)$. We can use the Morse lemma to write $F$ as a quadratic form in a neighborhood of $\left(1 / 2, x_{i}\right)$, which will be invariant under the action of the isotropy subgroup $G_{x_{i}}$. As the $S^{n}$ is a component of a level surface of $F$, it follows that $G_{x_{i}}$ acts orthogonally on the sphere. By choosing $\varepsilon$ small enough, we can ensure that the subgroup of $G$ which maps the sphere to itself is simply $G_{x_{i}}$.

In the following proof, we will need a technical proposition to the effect that the surgery can be done so that not only does the group action preserve the orientation of $V(1) \oplus W(1)$, but the isotropy groups preserve the individual orientations of the connected components of $V(1)$ and $W(1)$. We will show slightly more, namely,

Proposition 13. $X \cup(-X)$ can be equivariantly surgered to a disjoint union of spheres in such a way that at each surgery step, the isotropy group of a component of $V(1) \oplus W(1)$ fixes one of the factors in the product $D^{n+1-1} \times D^{i}$.

Proof. We will prove the proposition for continuous surgery; the construction can be smoothed out. To fix notation, let $D^{k}(\varepsilon)$ denote $\{v \in$ $\left.\mathbb{R}^{k}:|v| \leq \varepsilon\right\}$ and let $D^{k}$ denote $D^{k}(1)$. Then a solid $(k+1, n-k)$ $G$-handle $A_{\varepsilon}$ is defined to be

$$
A_{\varepsilon}=G \times_{H}\left(D^{k+1}(\varepsilon) \times D^{n-k}\right),
$$

and $A$ is defined by

$$
A=G \times{ }_{H}\left(D^{k+1} \times D^{n-k}\right),
$$

where $H$ acts by a diagonal action on $D^{k+1}(\varepsilon) \times D^{n-k}$, and orthogonally on each factor. $H$ will be called the isotropy group of $A$. Define $\partial_{+} A$ by

$$
\partial_{+} A=G \times{ }_{H}\left(S^{k} \times D^{n-k}\right) .
$$

Suppose that $B$ is an $(n+1)$-dimensional $G$-manifold and that $\partial_{*} B$ is an $n$-dimensional submanifold of $\partial B$. Given an embedding of $\partial_{+} A$ in $\partial B-\partial_{*} B$, a solid $(k+1, n-k) G$-handle extension $B^{\prime}$ of $B\left(\operatorname{rel} \partial_{*} B\right)$ 
is defined by

$$
B^{\prime}=B \bigcup_{\partial_{+} A} A
$$

Because $\partial_{*} B$ is embedded in $\partial B^{\prime}$, it makes sense to talk about a sequence of $G$-handle extensions of $B\left(\operatorname{rel} \partial_{*} B\right)$.

Suppose that $B_{0}$ is an $(n+1)$-dimensional submanifold of $B$ and takes $\partial_{*} B_{0}$ to be $\partial B_{0} \cap B$, an $n$-dimensional submanifold of both $\partial B_{0}$ and $\partial B$. Then a Morse theory argument shows that $B$ is given by a sequence of $G$-handle extensions of $B_{0}\left(\operatorname{rel} \partial_{*} B_{0}\right)$. For example, our total surgery is given by taking $B=X \times I$ and $B_{0}=\bigcup D^{n+1}$. Giving a representation of $B$ as a sequence of $G$-handle extensions of $B_{0}\left(\operatorname{rel} \partial_{*} B_{0}\right)$ is equivalent to giving a sequence of $G$-surgeries from $\partial B$ to $\partial B_{0}$, which do not touch $\partial_{*} B_{0}$.

Definition. A $(k+1, n-k) G$-handle $A$ is nice if the isotropy group $H$ acts trivially on $S^{k}$.

We wish to show that a sequence of $G$-handle extensions can be done by a sequence of nice $G$-handle extensions. It suffices to show this for a single $G$-handle extension, $B^{\prime}=B \cup_{\partial_{+} A} A$. (See Figure 1, next page, where we illustrate the case of $n=2, k=1$.) We will do this by induction on $|H|$. For $H$ trivial, there is nothing to show. Let us write $A$ as

$$
A=\left([1 / 2,1] \times \partial_{+} A\right) \cup_{\partial_{+} A_{1 / 2}} A_{1 / 2} \text {. }
$$

Let $B^{\prime \prime}$ be a disjoint union of $B$ and $A_{1 / 2}$ (Figure 2). Then $B^{\prime \prime}$ is a nice $(0, n+1) \quad G$-handle extension of $B$. Thus it suffices to show that $B^{\prime}$ is a nice $G$-handle extension of $B^{\prime \prime}$. This will be true if for small $\varepsilon,[1 / 2,1] \times$ $\partial_{+} A$ is given by a sequence of nice $G$-handle extensions of $([1 / 2,1 / 2+\varepsilon] \cup$ $[1-\varepsilon, 1]) \times \partial_{+} A$, which in turn will be true if $[1 / 2,1] \times\left(S^{k} \times D^{n-k}\right)$ is given by a sequence of nice $H$-handle extensions of $([1 / 2,1 / 2+\varepsilon] \cup$ $[1-\varepsilon, 1]) \times\left(S^{k} \times D^{n-k}\right)$. For notational simplicity, we will reparametrize and show that $C^{\prime}=[-\varepsilon, 1+\varepsilon] \times\left(S^{k} \times D^{n-k}\right)$ is given by a sequence of nice $H$-handle extensions of $C=([-\varepsilon, 0] \cup[1,1+\varepsilon]) \times\left(S^{k} \times D^{n-k}\right)$ (Figure 3).

The idea will be to do an explicit nice $H$-handle extension which includes the $H$-fixed point set and then to argue that the other extensions can be done with isotropy groups which are proper subgroups of $H$. Let $S^{j}$ denote $\left(S^{k}\right)^{H}$, the points of $S^{k}$ which are fixed by $H$. Write $S^{j}$ as a union of two hemispheres: $S^{j}=D_{+}^{j} \cup D_{-}^{j}$. Let $S^{j} \times D^{k-j}$ be an $H$ invariant tubular neighborhood of $S^{j}$ in $S^{k}$ (Figure 4, which illustrates 

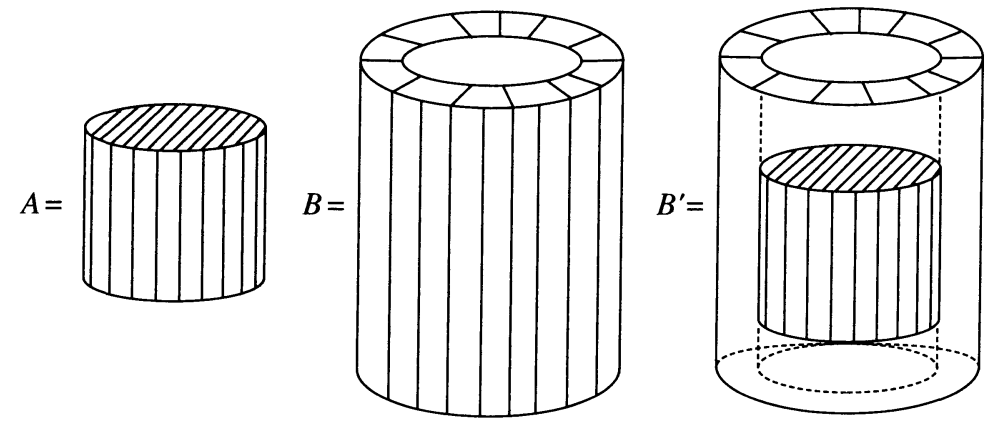

FIGURE 1

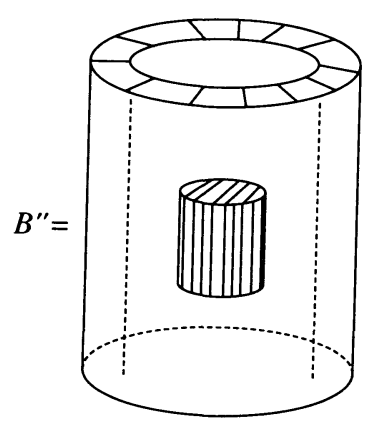

Figure 2

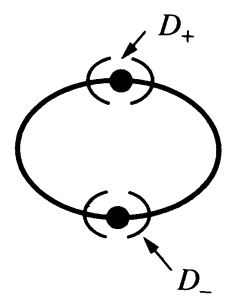

FIGURE 4

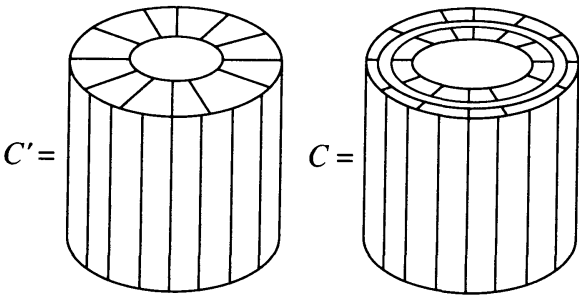

Figure 3

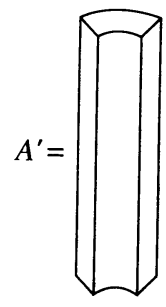

FIGURE 5

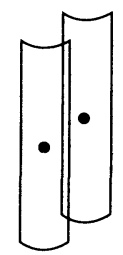

Figure 8

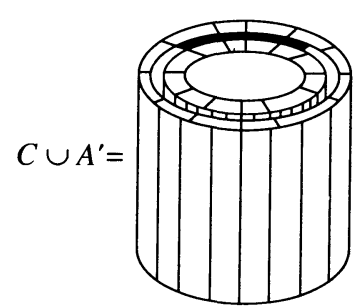

FIGURE 6

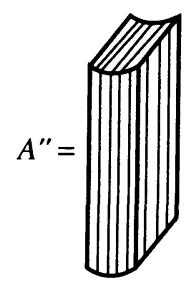

Figure 9 
the case $j=0)$. Put $A^{\prime}$ to be $[0,1] \times\left(D_{+}^{j} \times D^{k-j}\right) \times D^{n-k}$ (Figure 5), which has a natural embedding in $[0,1] \times S^{k} \times D^{n-k}$. Then $C \cup A^{\prime}$ is a nice $(1, n) H$-handle extension of $C$, which embeds in $C^{\prime}$ (Figure 6). As a set, $\partial\left(C \cup A^{\prime}\right)$ can be written as a disjoint union by

$$
\begin{aligned}
\partial\left(C \cup A^{\prime}\right)= & \left(\{-\varepsilon\} \times S^{k} \times D^{n-k}\right) \cup\left((-\varepsilon, 0) \times S^{k} \times S^{n-k-1}\right) \\
& \cup\left(\{0\} \times\left(S^{k}-\left(D_{+}^{j} \times D^{k-j}\right)\right) \times D^{n-k}\right. \\
& \cup\left((0,1) \times \partial\left(D_{+}^{j} \times D^{k-j} \times D^{n-k}\right)\right) \\
& \cup\left(\{1\} \times\left(S^{k}-\left(D_{+}^{j} \times D^{k-j}\right)\right) \times D^{n-k}\right) \\
& \cup\left((1,1+\varepsilon) \times S^{k} \times S^{n-k-1}\right) \cup\left(\{1+\varepsilon\} \times S^{k} \times D^{n-k}\right) .
\end{aligned}
$$

In particular, $\left(\{0\} \times D_{-}^{j}\right) \cup\left((0,1) \times S^{j-1}\right) \cup\left(\{1\} \times D_{-}^{j}\right)$ is a $j$-sphere $T^{j}$ which is embedded in $\partial\left(C \cup A^{\prime}\right)-\partial C$ and is pointwise fixed by $H$ (Figure 7). There is a tubular neighborhood of $T^{j}$ of the form $T^{j} \times D^{k-j} \times D^{n-k}$ in $\partial\left(C \cup A^{\prime}\right)-\partial C^{\prime}$ (Figure 8). This tubular neighborhood can be written in the form $\partial_{+} A^{\prime \prime}$, where $A^{\prime \prime}=D^{j+1} \times D^{k-j} \times D^{n-k}$ is embedded in $C^{\prime}$, with $D^{j+1}=[0,1] \times D_{-}^{j}$ (Figure 9). Thus, $C \cup A^{\prime} \cup A^{\prime \prime}$ is a nice $(j+1, n-j) H$-handle extension of $C \cup A^{\prime}$, which embeds in $C^{\prime}$ (Figure 10 , next page).

Now

$$
\begin{aligned}
C \cup A^{\prime} \cup A^{\prime \prime} & \\
= & \left([-\varepsilon, 0] \times S^{k} \times D^{n-k}\right) \cup\left([0,1] \times S^{j} \times D^{k-j} \times D^{n-k}\right) \\
& \cup\left([1,1+\varepsilon] \times S^{k} \times D^{n-k}\right) .
\end{aligned}
$$

Thus,

$$
C^{\prime}-\left(C \cup A^{\prime} \cup A^{\prime \prime}\right)=(0,1) \times\left(S^{k}-\left(S^{j} \times D^{k-j}\right)\right) \times D^{n-k}
$$

is uniformly bounded away from the fixed point set

$$
\left(C^{\prime}\right)^{H}=[-\varepsilon, 1+\varepsilon] \times S^{j} \times\left(D^{n-k}\right)^{H} \quad(\text { Figure 12). }
$$

That is, there exists a $\delta>0$ such that for all $x \in C^{\prime}-\left(C \cup A^{\prime} \cup A^{\prime \prime}\right)$ and all $h \in H, d(x, h x)>\delta$. Consider constructing $C^{\prime}$ from $C \cup A^{\prime} \cup A^{\prime \prime}$ by a sequence of $H$-handle extensions. Because the handles can be chosen to be arbitrarily small, it follows that we can choose them small enough that the isotropy group of a handle is a proper subgroup of $H$. Then, by induction, $C^{\prime}$ can be constructed from $C \cup A^{\prime} \cup A^{\prime \prime}$ by a sequence of nice $H$-handle extensions. q.e.d. 


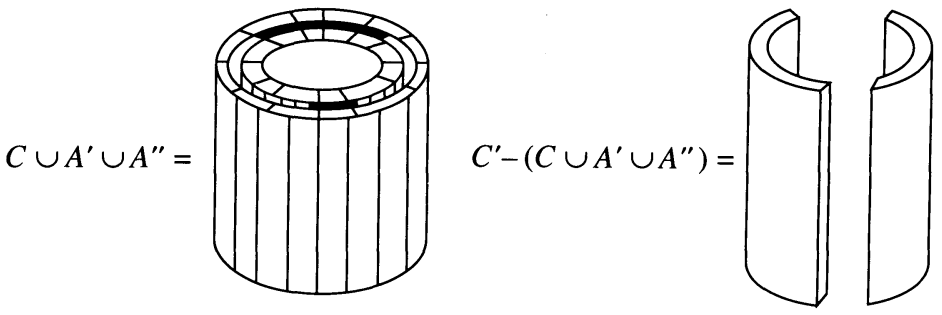

FIGURE 10

FIGURE 11

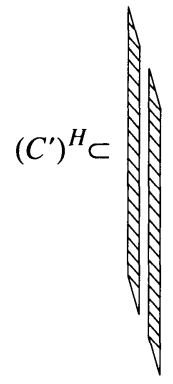

FIGURE 12

In order to show that $T$ equals $\tau$ in the case of a free action, the method used by Müller was to first show that for two representations $\rho_{1}$ and $\rho_{2}$, one has that

$$
T_{\rho_{1}}-T_{\rho_{2}}=\tau_{\rho_{1}}-\tau_{\rho_{2}}
$$

This reduces the problem to the case of trivial $\rho$, which is then handled by surgery methods. In our case, this first step is problematic. The difference of the zeta functions corresponding to $\rho_{1}$ and $\rho_{2}$ is not a priori finite, as in the case of free actions. This is because any element of $G$ can have a fixed point in its action on $X$, unlike the case of a free action. Thus we will skip the step of reducing to a trivial $\rho$ and instead do surgery directly for any $\rho$. The reduction to a trivial representation is used in [15] because there one was effectively dealing with a free action on a possibly noncompact space, with compact quotient. Here we only consider actions on compact spaces.

The second step of [15] is to form a combinatorial torsion $\tau^{c}$, defined using a triangulation. One then shows that under surgery, as the triangulation becomes infinitely fine, $\tau-\tau^{c}$ jumps by the same amount as $T-\tau^{c}$. This reduces the problem to a computation on spheres.

More specifically, define an inner product on $C^{*}(K, \rho)$ by $\left(a, a^{\prime}\right)=$ $\int_{X} W a \wedge W a^{\prime}$, there $W$ is the Whitney operator. Define $\delta^{c}$ to be the adjoint of $d^{c}$, and put

$$
\Delta^{c}=\delta^{c} d^{c}+d^{c} \delta^{c}
$$

\section{Definition.}

$$
\tau^{c}=\left.\frac{d}{d s}\right|_{s=0} \frac{1}{\Gamma(s)} \int_{0}^{\infty} T^{s-1} \operatorname{Tr}(-)^{F} F e^{-T\left(\Delta^{c}\right)^{\prime}} d T,
$$

where the prime denotes the omission of zero eigenvalues.

In order to describe the surgery operation, we use the notation of $\S 10$ of [15], but with everything generalized to the $G$-equivariant setting. The reader may wish to refer to that paper for details. For completeness, we 
will recall the basic setup. $X$ is a closed $n$-dimensional $G$-manifold, and $W_{1}, W_{2}$, and $W_{3}$ are $n$-dimensional $G$-submanifolds such that $X=$ $W_{1} \cup W_{2}, W_{3}=W_{1} \cap W_{2}$. Furthermore, $W_{2}$ is $G$-diffeomorphic to a $G$ bundle of the form $\partial V(1) \oplus W(1)$, which is topologically a disjoint union of $S^{p} \times D^{q+1}$,s, and $W_{3}$ is topologically a disjoint union of $S^{p} \times S^{q} \times$ $I$ 's, where $I=[-1,1]$. Thus $W_{2}$ is a $G$-handle, and $W_{3}$ is a tubular neighborhood of $\partial W_{2} . X^{\prime}$ is obtained surgically from $X$ by gluing to $W_{1}^{\prime}=W_{1}$ a $G$-handle $W_{2}^{\prime}$ which is a disjoint union of $D^{p+1} \times S^{q}$, s. $W_{3}^{\prime}=W_{1}^{\prime} \cap W_{2}^{\prime}$ is a tubular neighborhood of $\partial W_{2}^{\prime}$, and is again a disjoint union of $S^{p} \times S^{q} \times I$ 's. Thus $X^{\prime}$ is obtained from $X$ by removing a $G$-handle $W_{2}$ and gluing in a $G$-handle $W_{2}^{\prime}$. Let $M_{1}$ and $M_{2}$ denote the doubles of $W_{2}$ and $W_{2}^{\prime}$, so that $M$ is a disjoint union of $S^{p} \times S^{q+1}$,s, and $M_{2}$ is a disjoint union of $S^{p+1} \times S^{q}$ 's.

First, let us show that the de Rham map

$$
A: \Lambda^{q}(X) \otimes \mathbb{R}^{N} \rightarrow C^{q}(K) \otimes \mathbb{R}^{N}
$$

and the Whitney map

$$
W: C^{q}(K) \otimes \mathbb{R}^{N} \rightarrow \Lambda^{q}(X) \otimes \mathbb{R}^{N}
$$

can be restricted to maps between $\Lambda^{q}(X, \rho)$ and $C^{q}(K, \rho)$.

Proposition 14. $a\left(g^{-1}\right)^{*}$ commutes with $A$ and $W$.

Proof. Recall that for all $f \in \Lambda^{q}(X) \otimes \mathbb{R}^{N}, A f=\sum\left(\int_{\sigma} f\right) \sigma$, where the sum runs over $q$-simplices $\sigma$ of $X$, and $\sigma$ is also considered as an element of $C^{q}(K)$. Then

$$
\begin{aligned}
A a\left(g^{-1}\right)^{*} f & =\sum\left(\int_{\sigma} a\left(g^{-1}\right)^{*} f\right) \sigma=\sum\left(\int_{a\left(g^{-1}\right) \sigma} f\right) \sigma \\
& =\sum\left(\int_{\sigma^{\prime}} f\right) a(g) \sigma^{\prime}=a(g) \sum\left(\int_{\sigma^{\prime}} f\right) \sigma^{\prime}=a(g) A f .
\end{aligned}
$$

For the Whitney map, let us recall that for a $q$-simplex $\sigma=\left[p_{i_{0}}, p_{i_{1}}, \cdots\right.$, $p_{i_{q}}$ ] of $K, W$ is defined by

$$
\begin{aligned}
& W(\sigma)=q ! \sum_{k=0}^{q}(-1)^{k} \mu_{i_{k}} d \mu_{i_{0}} \bigwedge \cdots \bigwedge \widehat{d \mu_{i_{k}}} \bigwedge \cdots \bigwedge d \mu_{i_{q}} \text { if } q>0, \\
& W\left[p_{i}\right]=\mu_{i} \text { if } q=0
\end{aligned}
$$

where $\mu_{i}$ is the barycentric coordinate corresponding to the vertex $p_{i}$. For an element $c=\sum \sigma \otimes v_{\sigma}$ of $C^{q}(K) \otimes \mathbb{R}^{N}$, we define

$$
W c=\sum W(\sigma) \otimes v_{\sigma} \in \bigwedge^{q}(X) \otimes \mathbb{R}^{N}
$$


Then

$$
\begin{gathered}
W a\left(g^{-1}\right)^{*} c=W \sum a(g) \sigma \otimes v_{\sigma}=\sum W(a(g) \sigma) \otimes v_{\sigma} \\
a\left(g^{-1}\right)^{*} W c=a\left(g^{-1}\right)^{*} \sum W(\sigma) \otimes v_{\sigma}=\sum a\left(g^{-1}\right)^{*} W(\sigma) \otimes v_{\sigma} .
\end{gathered}
$$

Thus, it suffices to show that

$$
W(a(g) \sigma)=a\left(g^{-1}\right)^{*} W(\sigma) .
$$

However, the simplex $a(g) \sigma$ is simply $\left[a(g) p_{i_{0}}, \cdots, a(g) p_{i_{q}}\right]$, and the barycentric coordinate for the vertex $a(g) p_{i_{j}}$ is $a\left(g^{-1}\right)^{*} \mu_{i_{j}}$, from which the lemma follows. q.e.d.

One can now check that each equation and estimate of $\S 1-5$ of [15] goes through to our situation provided that one makes the following replacements:

1. Every $C^{q}\left(K, L_{\rho}\right)$ or $C^{q}(K)$ of [15] is replaced by $C^{q}(K, \rho)$.

2. Every $\Lambda^{q}\left(X, L_{\rho}\right)$ or $\Lambda^{q}(X)$ of [15] is replaced by $\Lambda^{q}(X, \rho)$.

3. Every trace over $C^{q}$ or $\Lambda^{q}$ of [15] is replaced by a trace over $C^{q}(K, \rho)$ or $\Lambda^{q}(X, \rho)$.

Note. If we had the special case of a free action of $G$ on $X$, then the space " $X$ " considered in [15] would be the space $X / G$ of the present paper. However, all of the estimates for " $X$ " also work for $X$.

As the last tool of machinery to prove the equality of $\tau$ and $T$, we need the equivariant generalization of the parametrices of [15]. Let $\left\{U_{\alpha}\right\}$ be a $G$-covering of $X$ by open sets with smooth boundary, i.e., for each $\alpha$ and each $g \in G, g U_{\alpha}$ is also an open set in the covering. Let $\left\{\varphi_{\alpha}\right\}$ be a partition of unity subordinate to $\left\{U_{\alpha}\right\}$ such that for each $\alpha$ and each $g \in G, a\left(g^{-1}\right)^{*} \varphi_{\alpha}$ is also in the set of partition functions. Let $\left\{\Psi_{\alpha}\right\}$ be an equivariant set of functions satisfying $\varphi_{\alpha} \Psi_{\alpha}=\varphi_{\alpha}$. Let $\Delta_{\alpha}^{q}$ be the Laplacian acting on $\mathbb{R}^{N}$ valued $q$-forms on $U_{\alpha}$ with absolute boundary conditions. Let $H_{\alpha}^{q}$ be the projection over $\operatorname{Ker} \Delta_{\alpha}^{q}$, and put

$$
D_{\alpha}^{q}=\Delta_{\alpha}^{q}+H_{\alpha}^{q}
$$

Finally, define the analytic parametrix to be

$$
E_{\alpha}^{q}(s)=\sum_{\alpha} \varphi_{\alpha}\left(D_{\alpha}^{q}\right)^{-s} \Psi_{\alpha}
$$

a parametrix on $\Lambda^{q}(x) \otimes \mathbb{R}^{N}$. 
For the combinatorial parametrix, let $K$ be a smooth $G$-triangulation of $X$, and let $\left\{U_{\alpha}\right\}$ be a $G$-covering of $X$ such that for each $\alpha, K$ induces a smooth triangulation $\left(K_{\alpha}, L_{\alpha}\right)$ of $\left(U_{\alpha}, \partial U_{\alpha}\right)$. Let $W_{\alpha}$ be the Whitney map with respect to $K_{\alpha}$, let $\Delta_{\alpha(c)}^{q}$ be the combinatorial Laplacian on $C^{q}\left(K_{\alpha}\right) \otimes \mathbb{R}^{N}$, let $H_{\alpha(c)}^{q}$ be the projection onto the kernel of $\Delta_{\alpha(c)}^{q}$, put

$$
D_{\alpha(c)}^{q}=\Delta_{\alpha(c)}^{q}+H_{\alpha(c)}^{q},
$$

and define the combinatorial parametrix to be

$$
E_{\alpha(c)}^{q}(s)=\sum_{\alpha} \varphi_{\alpha} W_{\alpha}\left(D_{\alpha(c)}^{q}\right)^{-s} A \psi_{\alpha}
$$

acting on $\Lambda^{q}(X) \otimes \mathbb{R}^{N}$.

One can now check that every equation and estimate of $\S 8$ of [15] goes through provided that the replacements $(*)$ are made.

As said above, we will not need the first comparison theorem of [15], which involves the difference of torsions for two different representations. We will show that under surgery (and as the size of the mesh goes to zero)

$$
T_{X}-T_{X^{\prime}}-\frac{1}{2} T_{M_{1}}+\frac{1}{2} T_{M_{2}}
$$

equals

$$
\tau_{X}^{c}-\tau_{X^{\prime}}^{c}-\frac{1}{2} \tau_{M_{1}}^{c}+\frac{1}{2} \tau_{M_{2}}^{c}
$$

and that this in turn equals

$$
\tau_{X}-\tau_{X^{\prime}}-\frac{1}{2} \tau_{M_{1}}+\frac{1}{2} \tau_{M_{2}}
$$

As the Riemannian metric on $X$ varies, we know how $T_{\rho}$ varies from Proposition 2 . The only way that $\tau_{\rho}$ varies is through the volume forms on the cohomology groups $H^{*}(K, \rho)$. This variation is given in Proposition $2^{\prime}$, and one has that $\tau_{\rho}$ varies in the same way as $T_{\rho}$. Thus $T_{\rho}-\tau_{\rho}$ is independent of the $G$-invariant Riemannian metric on $X$. Choose Riemannian metrics on $X$ and $X^{\prime}$ so that $W_{1} \subset X$ and $W_{1}^{\prime} \subset X^{\prime}$ are isometric, and $W_{3}$ and $W_{3}^{\prime}$ are isometric to a disjoint union of standard $S^{p} \times S^{q} \times I$ 's. Using an open covering adapted to the handle-bundle addition as in [15], we have

$$
\begin{aligned}
& \left.\operatorname{Tr} E_{q}(x)\right|_{\Lambda^{q}(X) \otimes \mathbb{R}^{N}}-\left.\operatorname{Tr} E_{q}(x)\right|_{\Lambda^{q}\left(X^{\prime}\right) \otimes \mathbb{R}^{N}} \\
& \quad=\left.\frac{1}{2} \operatorname{Tr} E_{q}(s)\right|_{\Lambda^{q}\left(M_{1}\right) \otimes \mathbb{R}^{N}}-\left.\frac{1}{2} \operatorname{Tr} E_{q}(s)\right|_{\Lambda^{q}\left(M_{2}\right) \otimes \mathbb{R}^{N}}
\end{aligned}
$$


The analogue of 8.44 of [15] is that $\zeta_{q}^{c}(s)-\operatorname{Tr} E_{q}^{c}(s)$ converges uniformly on compact subsets of $\mathbb{C}$ to $\zeta_{q}(s)-\operatorname{Tr} E_{q}(s)$ as the mesh size goes to zero. It follows that

$$
\tau_{X}^{c}-\tau_{X^{\prime}}^{c}-\frac{1}{2} \tau_{M_{1}}^{c}+\frac{1}{2} \tau_{M_{2}}^{c}
$$

converges to

$$
T_{X}-T_{X^{\prime}}-\frac{1}{2} T_{M_{1}}+\frac{1}{2} T_{M_{2}}
$$

as the mesh size goes to zero.

By the Mayer-Vietoris sequence for the decomposition of $X$ as $W_{1} \cup$ $W_{2}$, it follows as in [15] that

$$
\tau_{X}^{c}-\tau_{X^{\prime}}^{c}-\frac{1}{2} \tau_{M_{1}}^{c}+\frac{1}{2} \tau_{M_{2}}^{c}
$$

converges to

$$
\tau_{X}-\tau_{X^{\prime}}-\frac{1}{2} \tau_{M_{1}}+\frac{1}{2} \tau_{M_{2}}
$$

as the mesh size goes to zero.

We now have that

$$
(T-\tau)(X)-\left(T-\tau^{\prime}\right)\left(X^{\prime}\right)=\frac{1}{2}(T-\tau)\left(M_{1}\right)-\frac{1}{2}(T-\tau)\left(M_{2}\right) .
$$

In order to show that $T-\tau$ is invariant under a nice surgery, it suffices to show that it vanishes for a standard $G$-action on a disjoint union of odd-dimensional $S^{p} \times S^{q}$ 's. Finally, to show that $(T-\tau)(X)$ vanishes, by the handle-body decomposition it suffices to show that $T-\tau$ vanishes for a standard $G$-action on a disjoint union of odd-dimensional spheres. Both of these will follow from

Proposition 15. Suppose that $G$ acts on $X=\bigcup_{i=1}^{m} P_{i}$, where each $P_{i}$ is a product $S^{p} \times S^{q}$ of odd dimension. Let $H_{i}$ denote the subgroup which maps $P_{i}$ to itself. Suppose that for each element $h$ of $H_{i}$ the action of $h$ on $S^{p} \times S^{q}$ is given by a product of special orthogonal group actions. Then for all representations $\rho: G \rightarrow O(N), T_{\rho}(X)=\tau_{\rho}(X)$.

Proof. Let $\rho_{i}$ denote $\rho$ restricted to $H_{i}$. Because the heat kernel vanishes between $P_{i}$ and $P_{j}$ when $i \neq j$, we have $T_{\rho}(X)=\sum_{i} T_{\rho_{i}}\left(P_{i}\right)$, and similarly for $\tau_{\rho}$. Without loss of generality, suppose that $p$ is odd and $q$ is even. For any $h \in H_{i}$, we have that the Lefschetz number of $h$ acting on $S^{p}$ vanishes. Then it follows from Proposition 3 and Proposition 5 that

$$
T_{\rho_{i}}\left(P_{i}\right)=T_{\rho_{i} \otimes \rho_{H_{2}}}\left(S^{p}\right)
$$


and similarly for $\tau_{\rho_{i}}$. Thus it suffices to check the equality of $T$ and $\tau$ for special orthogonal actions on odd-dimensional spheres. This was done in Proposition 12. q.e.d.

We have now proved

Proposition 16. Suppose that a finite group $G$ acts by orientationpreserving diffeomorphisms on an odd-dimensional closed oriented manifold $X$. Then for any orthogonal representation $\rho$ of $G, T_{\rho}=\tau_{\rho}$.

\section{Nonequality of $T$ and $\tau$ for even-dimensional manifolds}

By Proposition 1, if $X$ is even-dimensional, then $T=0$. On the other hand, the calculations of $\S$ VI show that even for special orthogonal actions on spheres, $\tau$ is generally nonzero. For example, if $S^{p}$ is an evendimensional sphere, $S^{q}$ is an odd-dimensional sphere, and $\rho$ is an acyclic representation, then from (6.13) we have

$$
\tau_{\rho}\left(S^{p} \# S^{q}\right)=\tau_{\rho}\left(S^{p}\right)+\tau_{\rho}\left(S^{q}\right)-\tau_{\rho}\left(S^{p} \times S^{q}\right) .
$$

Because the Lefschetz number of a special orthogonal action on $S^{p}$ is two, and vanishes for a special orthogonal action on $S^{q}$, it follows that

$$
\begin{aligned}
\tau_{\rho}\left(S^{p} \# S^{q}\right) & =\tau_{\rho}\left(S^{p}\right)+\tau_{\rho}\left(S^{q}\right)-\left(2 \tau_{\rho}\left(S^{q}\right)+0 \tau_{\rho}\left(S^{p}\right)\right) \\
& =\tau_{\rho}\left(S^{p}\right)-\tau_{\rho}\left(S^{q}\right) .
\end{aligned}
$$

The action on $S^{q}$ and the representation $\rho$ can clearly be chosen in order to make $\tau\left(S^{p} \# S^{q}\right)$ nonzero.

One can understand the nonequality in the following way. The proof of Proposition 1 involves using the Hodge duality operator. The analogous method in the combinatorial case would involve comparing the torsion of a $G$-simplicial complex with that of its dual cell complex.

One can define the combinatorial torsion for a $G$-cell complex, but the problem is that given a triangularization $K$ of $X$, if the group action is such that the isotropy groups are not all the same, then the dual cell complex $K^{*}$ will not be a $G$-cell complex. One can see this in the following way: Suppose that $\sigma^{(a)}$ and $\sigma^{(b)}$ are an $a$-simplex and a $b$-simplex in $K$, with $\sigma^{(a)} \subset \sigma^{(b)}$, and that $a<b$. Suppose that $\sigma^{(a)}$ and $\sigma^{(b)}$ have isotropy groups $H_{1}$ and $H_{2}$ with $H_{1} \neq H_{2}$. Now $\sigma^{(a)}$ is also a vertex in the dual triangularization $\bar{K}$ of $K$, and it lies in the interior of the dual cell $\sigma^{(a)^{*}}$. However, the simplex $s$ of $\bar{K}$ consisting of the chain $\left[\sigma^{(a)}, \sigma^{(b)}\right]$ meets $\sigma^{(a)}$ in $\bar{K}$ and has an isotropy group different from 
that of $\sigma^{(a)}$. This is impossible if the dual cell complex is to form a $G$ cell complex [2]. Thus $K^{*}$ can only be a $G$-cell complex if all of the isotropy subgroups of $G$ are equal.

Recall that if $H^{*}(K, \rho)$ is nonvanishing, then we define the combinatorial torsion $\tau$ using the harmonic forms to give the volume forms on $\left\{H^{k}(K, \rho)\right\}$. We will first show that in the even-dimensional case, $\tau$ is actually independent of the Riemannian metric used in the definition, even if $H^{*}(K, \rho)$ is nonvanishing.

Proposition 17. Let $X$ be an n-dimensional closed oriented smooth manifold, with $n$ even, on which the finite group $G$ acts by orientationpreserving diffeomorphisms. Let $K$ be a G-triangulation of $X$. Let $\rho: G \rightarrow$ $\mathrm{O}(N)$ be an orthogonal representation of $G$. Then the combinatorial torsion $\tau_{\rho}$ is independent of the Riemannian metric $g$ used to define the volume forms on the cohomology groups $\left\{H^{*}(K, \rho)\right\}$.

Proof. Let $g(\varepsilon)$ be a smooth 1-parameter family of $G$-invariant metrics on $X$. From Proposition 2', we have

$$
\frac{d}{d \varepsilon} \tau_{\rho}=-\left.\operatorname{Tr}\right|_{\operatorname{Ker} \Delta} \Pi(-){ }^{F}\left(\frac{d}{d \varepsilon} *\right) *^{-1},
$$

where the notation is that of $\S I I$. Because $n$ is even, we have that

$$
*(-)^{F} *^{-1}=(-1)^{n}(-)^{F}=(-)^{F} \text {. }
$$

Also,

$$
0=\frac{d}{d \varepsilon}\left(*^{2}\right)=* \frac{d}{d \varepsilon} *+\left(\frac{d}{d \varepsilon} *\right) *
$$

Then

$$
\begin{aligned}
\frac{d}{d \varepsilon} \tau_{\rho} & =-\left.\operatorname{Tr}\right|_{\operatorname{Ker} \Delta} \Pi(-)^{F}\left(\frac{d}{d \varepsilon} *\right) *^{-1} \\
& =\left.\operatorname{Tr}\right|_{\operatorname{Ker} \Delta} \Pi(-)^{F} *^{-1}\left(\frac{d}{d \varepsilon} *\right) \\
& =\left.\operatorname{Tr}\right|_{\operatorname{Ker} \Delta} \Pi(-)^{F} *^{-1}\left(\frac{d}{d \varepsilon} *\right) *^{-1} * \\
& =\left.\operatorname{Tr}\right|_{\operatorname{Ker} \Delta} \Pi *(-)^{F} *^{-1}\left(\frac{d}{d \varepsilon} *\right) *^{-1} \\
& =\left.\operatorname{Tr}\right|_{\operatorname{Ker} \Delta} \Pi(-) \\
& =0 . \quad \text { q.e.d. }
\end{aligned}
$$

Following the method of proof of Proposition 16, we can now give an equation for $\tau_{\rho}$ in the even-dimensional case in terms of an equivariant 
surgical decomposition of $X \cup(-X)$, as, for example, one obtains from a $G$-invariant Morse function on $X$.

Proposition 18. Let $X$ be an n-dimensional closed oriented smooth manifold, with $n$ even, on which the finite group $G$ acts by orientationpreserving diffeomorphisms. Let $\rho: G \rightarrow \mathrm{O}(N)$ be an orthogonal representation of $G$. Let $f$ be a G-invariant Morse function on $X$. For each integer $i$ satisfying $0 \leq i \leq n$, let $\left\{x_{i, j}\right\}$ denote the critical points of $f$ with index $i$. Using the notation of $\S V I I$, suppose that the action of $G$ preserves the orientations on $V(1)$ and $W(1)$. Then

$$
\begin{aligned}
\tau_{\rho}(X)= & -\frac{1}{4} \sum_{i \text { odd }} \sum_{j} \tau_{\rho_{i, j}}\left(S^{n-i-1} \times S^{i+1}\right) \\
& +\frac{1}{4} \sum_{i \text { even }} \sum_{j} \tau_{\rho_{i, j}}\left(S^{n-i} \times S^{i}\right),
\end{aligned}
$$

where $\rho_{i, j}$ denotes the restriction of the representation $\rho$ to the isotropy group of the point $x_{i, j}$. (Note that the torsion of a product can be computed by Proposition $5^{\prime}$.)

Proof. By $\S$ VII, we have in general that under surgery

$$
\left(\tau_{X^{\prime}}-T_{X^{\prime}}\right)=\left(\tau_{X}-T_{X}\right)-\frac{1}{2}\left(\tau_{M_{1}}-T_{M_{1}}\right)+\frac{1}{2}\left(\tau_{M_{2}}-T_{M_{2}}\right) .
$$

As all of these spaces are even-dimensional, $T$ vanishes and so

$$
\tau_{X^{\prime}}=\tau_{X}-\frac{1}{2} \tau_{M_{1}}+\frac{1}{2} \tau_{M_{2}}
$$

If we now consider building $X \cup(-X)$ by surgery from a disjoint union of spheres, then initially one has the following torsion of the spheres:

$$
\sum_{j} \tau_{\rho_{n, j}}\left(S^{n}\right)=\frac{1}{2} \sum_{j} \tau_{\rho_{n, j}}\left(S^{0} \times S^{n}\right)
$$

Upon doing surgery on an orbit of critical points with index $i$, one has that $M_{1}$ is a disjoint union of $S^{n-i-1} \times S^{i+1}$ 's and that $M_{2}$ is a disjoint union of $S^{n-i} \times S^{i}$ s. Thus

$$
\begin{aligned}
2 \tau_{\rho}(X)= & \tau_{\rho}(X \cup(-X)) \\
= & \frac{1}{2} \sum_{j} \tau_{\rho_{n, j}}\left(S^{0} \times S^{n}\right)-\frac{1}{2} \sum_{i} \sum_{j} \tau_{\rho_{i, j}}\left(S^{n-i-1} \times S^{i+1}\right) \\
& +\frac{1}{2} \sum_{i \neq n} \sum_{j} \tau_{\rho_{i, j}}\left(S^{n-i} \times S^{i}\right) .
\end{aligned}
$$


As the Lefschetz number vanishes for an orientation-preserving action on a closed oriented odd-dimensional manifold, Proposition $3^{\prime}$ implies that the sum in the second term is effectively over $i$ odd. Similarly, the sum in the third term is effectively over $i$ even. Hence the proposition follows.

Proposition 19. Let $\left\{\mathscr{O}_{j}\right\}$ denote the orders of the elements of $G$.

(i) Let $\mathscr{M}$ denote the multiplicative subgroup of $\mathbb{Q}\left(\left\{\exp \left(2 \pi i / \mathscr{O}_{j}\right)\right\}\right)$ generated by the exponentials of the torsions for the actions of $\mathbb{Z}_{\mathscr{O}_{j}}$ on $S^{0}$ and $S^{1}$. With the hypotheses of Proposition $18, \exp (2|G| \tau)$ is the absolute value of an element of $\mathscr{M}$.

(ii) Suppose that $G$ is the cyclic group $\mathbb{Z}_{p}$. Let $\mathscr{M}^{\prime}$ be the multiplicative subgroup of $\mathbb{Q}(\exp (2 \pi i / p))$ generated by $\{1-\exp (2 \pi i k / p): 0<k<p\}$. With the hypotheses of Proposition 18 , if $H^{*}(X, \rho)$ vanishes, then $\exp \left(2 \tau_{\rho}\right)$ is the absolute value of an element of $\mathscr{M}$.

Proof. (i) By a theorem of Artin, if $c$ denotes the character of the representation $\rho$, we have that $|G| c$ is an integer sum of characters of representations induced from cyclic subgroups of $G$ [11]. From the results of Propositions 18 and $5^{\prime}$, the fact that the Lefschetz number of a special orthogonal action on an even dimensional sphere is 2 , and the fact that $\tau_{\rho}$ is linear in the character of $\rho$, it suffices to prove that for a cyclic $\mathbb{Z}_{p}$ special orthogonal action on a sphere, the exponential of the torsion is the absolute value of an element of $\mathscr{M}$. As in Proposition 10, we can write the action as a join of actions on $S^{1}$ and $S^{0}$. (6.13) now applies. Unlike in the proof of Proposition 10, we must take some care in considering the possible occurrence of nontrivial real cohomology groups. The same volume forms for the cohomology groups $H^{*}(X \# Y, \rho), H^{*}(X, \rho), H^{*}(Y, \rho)$, and $H^{*}(X \# Y, X \cup Y, \rho) \cong H^{*}\left(S(X \times Y), p_{0} \cup p_{1}, p\right) \cong H^{*-1}(X \times Y, \rho)$ are used to define the torsions $\tau_{\rho}(X \# Y), \tau_{\rho}(X), \tau_{\rho}(Y)$, and $\tau_{\rho}(X \times Y)$, and the torsion $\tau(\mathscr{H})$ of the cohomology exact sequence. Let us pick Riemannian metrics on $X \# Y, X, Y$, and $X \times Y$ so that under the de Rham isomorphism, the generators of the integer cohomology of these spaces give an orthonormal basis for the real cohomology; because all of these spaces are spheres or products of spheres, this can be done. By Proposition 17, the result for $\tau_{\rho}(X \# Y)$ can be computed using these metrics without loss of generality. It follows from (1.4) of [4] that $\tau(\mathscr{H})=0$.

(ii) Using the arguments of the proof of (i), it suffices to show that a $\mathbb{Z}_{p}$ action on $S^{1}$ or $S^{0}$ with a nontrivial representation, $\tau_{\rho}$ is the absolute value of an element of $\mathscr{M}^{\prime}$. This follows from Proposition 9. q.e.d. 
As the result of Proposition 18 is purely topological, one would expect that there is also a proof which does not involve the analysis of $\S \mathrm{VI}$. We now give such a proof.

Proof of Proposition 18 (\#2). Let us start with a general framework for defining torsion. Let

$$
\stackrel{\partial}{\rightarrow} C_{-1} \stackrel{\partial}{\rightarrow} C_{0} \stackrel{\partial}{\rightarrow} C_{1} \stackrel{\partial}{\rightarrow} \cdots
$$

be a complex of real finite-dimensional vector spaces, each with a preferred basis. Suppose that there exists a positive integer $k$ such that if $|n|>k$, then $C_{n}=0$. We also assume that there is a preferred basis for $\left\{H_{n}(C)\right\}$.

The isomorphism classes of such complexes form an abelian monoid under direct sum. We will turn this into an abelian group in the usual way, which we refer to as $\mathscr{A}$.

There are four basic operations on $\mathscr{A}: H,-, S$, and $*$, which are defined as follows:

$$
\begin{array}{r}
(H(C))_{n}=H_{n}(C), \begin{array}{l}
\text { with the zero boundary map, and the } \\
\text { given preferred basis for } H_{n}(C),
\end{array} \\
(-C)_{n}=C_{n}, \quad \begin{array}{l}
\text { with the boundary map }-\partial \text { and the } \\
\text { same preferred bases for } C_{n} \text { and } H_{n}(C),
\end{array} \\
(* C)_{n}=\operatorname{Hom}\left(C_{-n}, \mathbb{R}\right), \quad \begin{array}{l}
\text { with boundary map being the } \\
\text { preferred bases, and })_{n}=C_{n-1}, \quad \text { and the dual bases } \\
\text { for Hom }\left(C_{-n}, \mathbb{R}\right) \text { and } H(* C)_{n} \\
\\
=\operatorname{Hom}\left(H_{-n}(C), \mathbb{R}\right) .
\end{array}
\end{array}
$$

These operations satisfy the relations

$$
\begin{aligned}
& -^{2}=*^{2}=\text { Identity, } H^{2}=H,-H=H-,-S=S-, \\
& -*=*-, H S=S H, H *=* H, S *=*\left(S^{-1}\right) .
\end{aligned}
$$

Let $\mathscr{B}$ denote the algebra of operators on $\mathscr{A}$ generated by the operations.

The torsion [14] of a complex gives a homomorphism $\mathscr{T}: \mathscr{A} \rightarrow \mathbb{R}$ (we are using the logarithmic form of the torsion). We have that

$$
\begin{aligned}
& \mathscr{T}(H(C))=0, \quad \mathscr{T}(-C)=\mathscr{T}(C), \quad \mathscr{T}(S(C))=-\mathscr{T}(C), \\
& \mathscr{T}(* C)=-\mathscr{T}(C) .
\end{aligned}
$$

If we define a homomorphism $\lambda: \mathscr{B} \rightarrow \operatorname{End}(\mathbb{R})$ by putting

$$
\lambda(H)=0, \quad \lambda(-)=1, \quad \lambda(*)=\lambda(S)=-1,
$$

then $\mathscr{T}$ is a $\lambda$-homomorphism. 
Given a chain map $f: C \rightarrow \bar{C}$, let us define a new complex $C(f)$ by putting

$$
C(f)_{n}=C_{n} \oplus \bar{C}_{n-1},
$$

with boundary operator

$$
\partial(c, \bar{c})=(\partial c, f(c)-\partial \bar{c}) .
$$

There are preferred bases for $\left\{C(f)_{n}\right\}$, although there are no preferrred bases for $H_{*}(C(f))$. We have an exact sequence

$$
0 \rightarrow-S(\bar{C}) \rightarrow C(f) \rightarrow C \rightarrow 0
$$

and the connecting map in the corresponding homology exact sequence is $H_{*}(f)$. Thus $H_{*}(f)$ is an isomorphism if and only if the complex $C(f)$ is acyclic, in which case $C(f) \in \mathscr{A}$.

Let us assume hereafter that $H_{*}(f)$ is an isomorphism. From Theorem 3.2 of [14], we have

$$
\mathscr{T}(C(f))=\mathscr{F}(C)-\mathscr{F}(\bar{C})+\mathscr{T}(\mathscr{H}),
$$

where $\mathscr{H}$ is the homology sequence arising from the exact sequence (8.27). Note that because the preferred basis of $C(f)$ only depends on those of $C$ and $\bar{C}$, the dependences of $\mathscr{T}(C), \mathscr{T}(\bar{C})$, and $\mathscr{T}(\mathscr{H})$ on the preferred bases of $H_{*}(C)$ and $H_{*}(\bar{C})$ cancel out on the right-hand side of (8.28).

Definition. A homology embedding is a chain map $j: H(C) \rightarrow C$ such that

$$
H_{*}(j): H_{*}(H(C))\left(=H_{*}(C)\right) \rightarrow H_{*}(C)
$$

is the identity map.

For a homology embedding, we have

$$
\mathscr{T}(C(j))=\mathscr{T}(H(C))-\mathscr{T}(C)+\mathscr{T}(\mathscr{H}) .
$$

As the connecting map in $\mathscr{H}$ is the identity map on $H_{*}(C), \mathscr{T}(\mathscr{H})=0$. We know that $\mathscr{T}(H(C))=0$, and so $\mathscr{T}(C(j))=-\mathscr{T}(C)$.

Definition. Given $C, \bar{C} \in \mathscr{A}$ and a chain map $f: C \rightarrow \bar{C}$ which is a homology isomorphism, define $\mathscr{T}(f)$ to be $\mathscr{T}(C(f))$.

Given $f$, there are induced chain maps

$$
\begin{aligned}
& H(f): H(C) \rightarrow H(\bar{C}), \quad-f:-C \rightarrow-\bar{C}, \\
& S(f): S(C) \rightarrow S(\bar{C}), \quad * f: * C \rightarrow * \bar{C} .
\end{aligned}
$$

These have torsions

$$
\begin{aligned}
& \mathscr{T}(H(f))=0, \quad \mathscr{T}(-f)=\mathscr{T}(f), \quad \mathscr{T}(S(f))=-\mathscr{T}(f), \\
& \mathscr{T}(* f)=-\mathscr{T}(f) .
\end{aligned}
$$


Furthermore, if $f: C \rightarrow \bar{C}$ and $g: \bar{C} \rightarrow \widetilde{C}$ are homology isomorphisms, then $\mathscr{T}(g \circ f)=\mathscr{T}(g)+\mathscr{T}(f)$.

Now let us consider the case where we have a Poincare duality map.

Definition. A $k$-dimensional Poincare duality map is a chain map $P: C \rightarrow S^{k}(* C)$ which induces a homology isomorphism $H P: H(C) \rightarrow$ $S^{k}(* H(C))$. Note that $P$ need not be an isomorphism of based chain complexes.

Let $j: H(C) \rightarrow C$ be a homology embedding. Then we can define a homology embedding

$$
j^{*}: S^{k}(* H(C)) \rightarrow S^{k}(* C)
$$

by requiring that the diagram

$$
H(C) \stackrel{H P}{\longrightarrow} S^{k}(* H(C))
$$

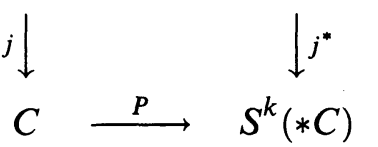

commute. It follows that

$$
\mathscr{T}\left(j^{*}\right)+\mathscr{T}(H P)=\mathscr{T}\left(j^{*}\right)=\mathscr{T}(P)+\mathscr{T}(j) .
$$

Also

$$
\mathscr{T}(j)=-\mathscr{T}(C)
$$

and

$$
\mathscr{T}\left(j^{*}\right)=-\mathscr{T}\left(S^{k}(* C)\right)=(-1)^{k} \mathscr{T}(C) .
$$

Thus,

$$
\mathscr{T}(P)=(-1)^{k} \mathscr{T}(C)+\mathscr{T}(C) .
$$

If $k$ is odd, then we conclude that $\mathscr{T}(P)=0$. On the other hand, if $k$ is even, then

$$
\mathscr{T}(C)=\frac{1}{2} \mathscr{T}(P) .
$$

Let us apply these equations to the setup of $\S \mathrm{IV}$, where $C$ is the complex of $\rho$-twisted (absolute or relative) cochains of a simplicial complex $K$. Suppose that $K$ is the triangulation of a closed oriented manifold $X$ of dimensional $k$ with a $G$-action, and that $K=K_{1} \cup K_{2}$, where $K_{1}$ and $K_{2}$ are of codimension zero, and with boundary. Suppose that $K_{1} \cap K_{2}=$ $B \times I$, where $B=\partial K_{1}=\partial K_{2}$. If we put $W_{i}=\bar{K}-K_{i}$, then $W_{1} \cap W_{2}=\varnothing$, and there are excision maps $\left(X, W_{i}\right) \cong\left(X_{i}, \partial X_{i}\right)$. 
We have a commutative diagram

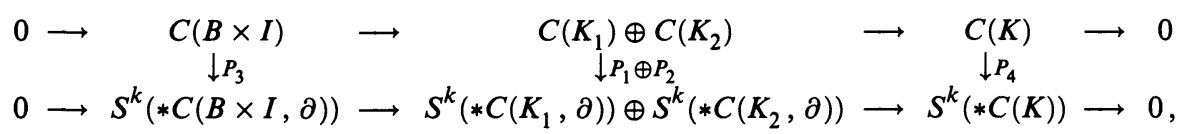
where the horizontal lines are exact. It follows that

$$
\mathscr{T}\left(P_{1}\right)+\mathscr{T}\left(P_{2}\right)=\mathscr{T}\left(P_{3}\right)+\mathscr{T}\left(P_{4}\right) .
$$

We also have a commutative diagram

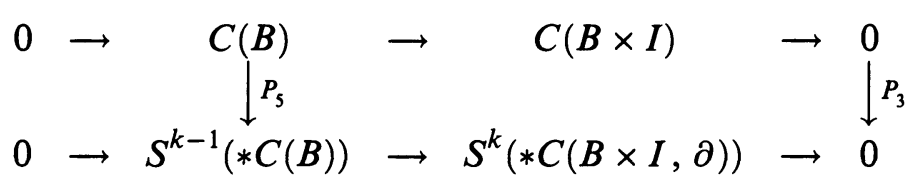

which gives that $\mathscr{T}\left(P_{3}\right)=\mathscr{T}\left(P_{5}\right)$. Thus

$$
\mathscr{T}\left(P_{1}\right)+\mathscr{T}\left(P_{2}\right)=\mathscr{T}\left(P_{4}\right)+\mathscr{T}\left(P_{5}\right) .
$$

If $k(=\operatorname{dim} X)$ is odd, then $\mathscr{T}\left(P_{4}\right)=0$ and so

$$
\mathscr{T}\left(P_{1}\right)+\mathscr{T}\left(P_{2}\right)=\mathscr{T}\left(P_{5}\right) \text {. }
$$

On the other hand, if $k$ is even, then $\mathscr{T}\left(P_{5}\right)=0$ and

$$
\mathscr{T}\left(P_{1}\right)+\mathscr{T}\left(P_{2}\right)=\mathscr{T}\left(P_{4}\right) \text {. }
$$

Let us now restrict to the case of $k$ even. In order to do a surgery operation, let us consider the case where $K_{2}$ is a $G$-invariant disjoint union of $S^{m} \times D^{m^{\prime}}$ 's, and another pair of simplicial complexes is given by putting $K_{2}^{\prime}=\bigcup D^{m} \times S_{m^{\prime}}, K^{\prime}=K_{1} \cup K_{2}^{\prime}$. Let us also put $K^{\prime \prime}$ to be the double of $K_{2}$ and $K^{\prime \prime \prime}$ to be the double of $K_{2}^{\prime}$, with duality maps $P_{4}^{\prime \prime}$ and $P_{4}^{\prime \prime \prime}$. Then

$$
\begin{aligned}
& \mathscr{T}\left(P_{4}\right)=\mathscr{T}\left(P_{1}\right)+\mathscr{T}\left(P_{2}\right), \\
& \mathscr{T}\left(P_{4}^{\prime}\right)=\mathscr{T}\left(P_{1}\right)+\mathscr{T}\left(P_{2}^{\prime}\right), \\
& \mathscr{T}\left(P_{4}^{\prime \prime}\right)=2 \mathscr{T}\left(P_{2}\right), \\
& \mathscr{T}\left(P_{4}^{\prime \prime \prime}\right)=2 \mathscr{T}\left(P_{2}^{\prime}\right) .
\end{aligned}
$$

Thus,

$$
\mathscr{T}\left(P_{4}\right)-\mathscr{T}\left(P_{4}^{\prime}\right)=\frac{1}{2}\left(\mathscr{T}\left(P_{4}^{\prime \prime}\right)-\mathscr{T}\left(P_{4}^{\prime \prime \prime}\right)\right) .
$$

It follows that

$$
\mathscr{T}(K)-\mathscr{T}\left(K^{\prime}\right)=\frac{1}{2}\left(\mathscr{T}\left(K^{\prime \prime}\right)-\mathscr{T}\left(K^{\prime \prime \prime}\right)\right) .
$$


Once we have this formula for how $\mathscr{T}$ changes under surgery, the rest of the proof of Proposition 18 follows as before. q.e.d.

Using (8.44) for the behavior of $\tau$ under unions, we can also give an inductive procedure to calculate $\tau$ in terms of fixed point sets, and the behavior of the group action in neighborhoods of these sets.

Proposition 20. Assume the hypotheses of Proposition 18. Let $H$ be a maximal isotropy subgroup of $G$. Let $F$ denote $\bigcup_{g} F i x\left(\mathrm{Hg}^{-1}\right)$, which is the union of the fixed point sets of the conjugates of $H, n(i)$ the dimension of a component $F_{i}$ of $F, N$ a G-invariant normal neighborhood of $F$, $D(N)$ the double of $N$, and $D(X \backslash N)$ the double of the complement of $N$. Then over $F_{i}, D(N)$ is an $S^{n-n(i)}$ bundle. Let $\rho_{i}$ denote the restriction of the representation $\rho$ to $\operatorname{Isotr}\left(F_{i}\right)$. Then

$$
\tau_{\rho}(X)=\sum_{i} \chi\left(F_{i}\right) \tau_{\rho_{i}}\left(S^{n-n(i)}\right)+\frac{1}{2} \tau_{\rho}(D(X \backslash N)) .
$$

Proof. From (8.44), we have that

$$
\tau_{\rho}(X)=\frac{1}{2} \tau_{\rho}(D(N))+\frac{1}{2} \tau_{\rho}(D(X \backslash N)) .
$$

We will compute $\tau_{\rho}(D(N))$ using Proposition 18. It suffices to do this separately for each component of $F$, and so we may assume that $F$ is connected. Let $f_{2}$ be a function on $D(N)$ whose restriction to any fiber is the standard height function on the sphere, with critical points at where the sphere intersects the two copies of $F$ that lie in $D(N)$. (Because $G$ acts orthogonally on the fibers of $N, f_{2}$ can be defined globally on $D(N)$.) Let $f_{3}$ be a Morse function on $F$, let $\pi$ denote the projection of $D(N)$ to $F$, and set $f_{1}$ to be $f_{2}+\pi^{*} f_{3}$. Then $f_{1}$ is a Morse function on $D(N)$, and to each critical point of $f_{3}$ of index $i$, there corresponds two critical points of $f_{1}$, with indices $i$ and $i+n-n^{\prime}$.

First, let us consider the case that $n^{\prime}$ is even. From Proposition 18, we have

$$
\begin{aligned}
\tau_{\rho}(D(N)) & \\
= & -\frac{1}{4} \sum_{i \text { odd }} \sum_{j}\left[\tau_{\rho_{i, j}}\left(S^{n-i-1} \times S^{i+1}\right)+\tau_{\rho_{i, j}}\left(S^{n^{\prime}-i-1} \times S^{n-n^{\prime}+i+1}\right)\right] \\
& +\frac{1}{4} \sum_{i \text { even }} \sum_{j}\left[\tau_{\rho_{i, j}}\left(S^{n-i} \times S^{i}\right)+\tau_{\rho_{i, j}}\left(S^{n^{\prime}-i} \times S^{n-n^{\prime}+i}\right)\right],
\end{aligned}
$$

where the sum on $i$ is from 0 to $n$. (Recall that $\tau_{\rho_{i, j}}$ denotes the torsion for the action of the isotropy group $H_{i, j}$ of $x_{i, j}$. $)$ As $H_{i, j}$ fixes the 
component of $F$ containing $x_{i, j}$ and the torsion vanishes for a trivial action in even dimensions, the product formula gives

$$
\begin{aligned}
\tau_{\rho}(D(N))= & -\frac{1}{2} \sum_{i \text { odd }} \sum_{j}\left[\tau_{\rho_{i, j}}\left(S^{n-i-1}\right)+\tau_{\rho_{i, j}}\left(S^{n-n^{\prime}+i+1}\right)\right] \\
& +\frac{1}{2} \sum_{i \text { even }} \sum_{j}\left[\tau_{\rho_{i, j}}\left(S^{n-i}\right)+\tau_{\rho_{i, j}}\left(S^{n-n^{\prime}+i}\right)\right] .
\end{aligned}
$$

By (8.2), each torsion in the above equation is the same as the torsion of the $H$-action on $S^{n-n^{\prime}}$. Thus,

$$
\begin{aligned}
\tau_{\rho}(D(N)) & =-\sum_{i \text { odd }} \sum_{j} \tau_{\rho^{\prime}}\left(S^{n-n^{\prime}}\right)+\sum_{i \text { even }} \sum_{j} \tau_{\rho^{\prime}}\left(S^{n-n^{\prime}}\right) \\
& =\chi(F) \tau_{\rho^{\prime}}\left(S^{n-n^{\prime}}\right) .
\end{aligned}
$$

Now let us suppose that $n^{\prime}$ is odd. Then by the same arguments,

$$
\begin{aligned}
\tau_{\rho}(D(N)) \\
=-\frac{1}{4} \sum_{i \text { odd }} \sum_{j}\left[\tau_{\rho_{i, j}}\left(S^{n-i-1} \times S^{i+1}\right)-\tau_{\rho_{i, j}}\left(S^{n^{\prime}-i} \times S^{n-n^{\prime}+i}\right)\right] \\
+\frac{1}{4} \sum_{i \text { even }} \sum_{j}\left[\tau_{\rho_{i, j}}\left(S^{n-i} \times S^{i}\right)-\tau_{\rho_{i, j}}\left(S^{n^{\prime}-i-1} \times S^{n-n^{\prime}+i+1}\right)\right] \\
=-\frac{1}{2} \sum_{i \text { odd }} \sum_{j}\left[\tau_{\rho_{i, j}}\left(S^{n-i-1}\right)-\tau_{\rho_{i, j}}\left(S^{n-n^{\prime}+i}\right)\right] \\
+\frac{1}{2} \sum_{i \text { even }} \sum_{j}\left[\tau_{\rho_{i, j}}\left(S^{n-i}\right)-\tau_{\rho_{i, j}}\left(S^{n-n^{\prime}+i+1}\right)\right]
\end{aligned}
$$

which equals, in consequence of (8.2),

$$
\begin{aligned}
& -\frac{1}{2} \sum_{i \text { odd }} \sum_{j}\left[\tau_{\rho^{\prime}}\left(S^{n-n^{\prime}}\right)-\tau_{\rho^{\prime}}\left(S^{n-n^{\prime}}\right)\right] \\
& +\frac{1}{2} \sum_{i \text { even }} \sum_{j}\left[\tau_{\rho^{\prime}}\left(S^{n-n^{\prime}}\right)-\tau_{\rho^{\prime}}\left(S^{n-n^{\prime}}\right)\right] \\
& =0 .
\end{aligned}
$$

Because $\chi(F)=0$ in this case, the proposition is still true.

\section{Orientation-reversing actions, manifolds with boundary}

Suppose that $G$ is a finite group that acts on a smooth oriented closed manifold $X$ and that some of the group elements reverse the orientation. 
Instead of trying to compare $\tau_{\rho}$ and $T_{\rho}$ directly, we will instead compare the functions $t$ and $t^{\prime}$ on $G$. We have already seen that if a group element $G$ preserves orientation, then $t(g)=t^{\prime}(g)$ for odd-dimensional $X$, while $t(g)$ can differ from $t^{\prime}(g)$ for even-dimensional $X$. The following propositions give what happens if $g$ reverses orientation.

Proposition 21. Suppose that the action of a group element $g$ reverses orientation. If $X$ is even-dimensional, then $t(g)=t^{\prime}(g)$.

Proof. Consider the action of $\mathbb{Z}_{2}=\{1, r\}$ on $S^{1}$, where $r\left(e^{i \theta}\right)=$ $e^{-i \theta}$. Then $(g, r) \in G \times \mathbb{Z}_{2}$ acts on the odd-dimensional manifold $X \times S^{1}$ in an orientation-preserving way. It is straightforward to see that product equation (3.2) for $t(g, r)$ continues to hold, along with the equation $\left(3.2^{\prime}\right)$ for $t^{\prime}(g, r)$. We know that $t(g, r)$ equals $t^{\prime}(g, r)$, and so

$$
0=\left(t-t^{\prime}\right)(g) L(r)+L(g)\left(t-t^{\prime}\right)(r) .
$$

However, $L(g)$ is zero and $L(r)=2$. Thus $t(g)=t^{\prime}(g)$.

Proposition 22. Suppose $\mathbb{Z}_{2}=\{1, r\}$ acts on an even-dimensional manifold in such a way that $r$ reverses orientation. Then for any orthogonal representation $\rho$ of $\mathbb{Z}_{2}, T_{\rho}=\tau_{\rho}$.

Proof. From Proposition 21, $t(r)=t^{\prime}(r)$. From the Cheeger-Müller equality of the standard analytic torsion with the Reidemeister torsion, it follows that $t(1)=t^{\prime}(1)$. Thus $T_{\rho}$ equals $\tau_{\rho}$. q.e.d.

Let us note that in [6] the equality of $T_{\rho}$ and $\tau_{\rho}$ was verified computationally for certain $\mathbb{Z}_{2}$ actions on Riemann surfaces.

Proposition 23. Suppose that the action of a group element $g$ reverses orientation. If $X$ is odd-dimensional, then $t(g)=0$.

Proof. The proof is the same as that of Proposition 1. The only difference is that now $a\left(g^{-1}\right)^{*}$ anticommutes with the Hodge duality operator $*$.

Proposition 24. There exist finite group actions on odd-dimensional closed orientable manifolds with elements $g$ that reverse orientation and satisfy $t(g) \neq t^{\prime}(g)$.

Proof. Using the join operation, one can construct cyclic group actions on spheres with elements $g$ satisfying $t^{\prime}(g) \neq 0$. q.e.d.

Let us apply the above results on orientation-reversing actions to discuss the torsion of a manifold with boundary. (See [13] for similar results.) Let $X$ be an oriented compact smooth manifold with boundary, with a Riemannian metric which is isometrically a product near the boundary. Let $D X$ denote the double of $X$, equipped with a Riemannian metric 
for which the involution $R$ acts by an isometry. Suppose that a finite group $G$ acts on $X$ by orientation-preserving diffeomorphisms. Then there is an induced action of $G \times \mathbb{Z}_{2}$ on $D X$. Let $t_{X}(g)$ denote the analytic torsion of the group element $g$, defined using differential forms on $X$ satisfying absolute boundary conditions [9]. Let $t_{X, \partial X}(g)$ denote the analytic torsion of the group element $g$, defined using differential forms on $X$ satisfying relative boundary conditions [15]. Define $T_{\rho}(X)$ and $T_{\rho}(X, \partial X)$ similarly.

Proposition 25. Suppose $\rho: G \rightarrow \mathrm{O}(N)$ is a representation such that the twisted cochains are acyclic on $X,(X, \partial X)$, and $\partial X$. If $X$ is evendimensional, then $T_{\rho}(X)=-T_{\rho}(X, \partial X)=T_{\rho}(\partial X) / 2$.

Proof. Note that in the equation defining the analytic torsion of $D X$, we can separate the differential forms into those which are even under the involution $R$ and those which are odd under $R$. This gives

$$
\begin{aligned}
& t_{D X}(g, 1)=t_{X}(g)+t_{X, \partial X}(g), \\
& t_{D X}(g, R)=t_{X}(g)-t_{X, \partial X}(g) .
\end{aligned}
$$

From Proposition 1, we have that $t_{D X}(g, 1)$ vanishes, and so

$$
t_{X, \partial X}(g)=-t_{X}(g) \text {. }
$$

(This can also be seen from Hodge duality.) The analogues of (9.2) and (9.3) for $\tau$ are [13]

$$
\begin{aligned}
& t_{D X}^{\prime}(g, 1)=t_{X}^{\prime}(g)+t_{X, \partial X}^{\prime}(g)+c \chi_{\partial X}(g), \\
& t_{D X}^{\prime}(g, R)=t_{X}^{\prime}(g)-t_{X, \partial X}^{\prime}(g)+c \chi_{\partial X}(g)
\end{aligned}
$$

for some constant $c$, whose exact value will not be important for us. From Proposition 21, it follows that

$$
t_{D X}(g, R)=t_{D X}^{\prime}(g, R) .
$$

Thus,

$$
2 t_{X}(g)=t_{X}^{\prime}(g)-t_{X, \partial X}^{\prime}(g)+c \chi_{\partial X}(g)
$$

and so

$$
2 T_{\rho}(X)=\tau_{\rho}(X)-\tau_{\rho}(X, \partial X) .
$$

From the equation for the torsion of an exact sequence of chain complexes [14], we have

$$
\tau_{\rho}(X)=\tau_{\rho}(X, \partial X)+\tau_{\rho}(\partial X)
$$


and so

$$
2 T_{\rho}(X)=\tau_{\rho}(\partial X)
$$

By Proposition 16, $\tau_{\rho}(\partial X)=T_{\rho}(\partial X)$, which proves the proposition.

Proposition 26. Suppose $\rho: G \rightarrow \mathrm{O}(N)$ is a representation such that the twisted cochains are acyclic on $X,(X, \partial X)$, and $\partial X$. If $X$ is odddimensional, then

$$
T_{\rho}(X)=\tau_{\rho}(X)-\tau_{\rho}(\partial X) / 2=T_{\rho}(X, \partial X)=\tau_{\rho}(X, \partial X)+\tau_{\rho}(\partial X) / 2
$$

Proof. Since now $t_{D X}(g, R)$ vanishes, we have

$$
t_{X, \partial X}(g)=t_{X}(g) \text {. }
$$

From Proposition 16, it follows that

$$
t_{D X}(g, 1)=t_{D X}^{\prime}(g, 1) .
$$

Then (9.2), (9.5), (9.12), and (9.13) give

$$
2 t_{X}(g)=t_{X}^{\prime}(g)+t_{X, \partial X}^{\prime}(g)+c \chi_{\partial X}(g),
$$

and so

$$
2 T_{\rho}(X)=\tau_{\rho}(X)+\tau_{\rho}(X, \partial X) .
$$

Using (9.10) we obtain

$$
2 T_{\rho}(X)=2 \tau_{\rho}(X)-\tau_{\rho}(\partial X)
$$

and

$$
2 T_{\rho}(X, \partial X)=2 T_{\rho}(X)=2 \tau_{\rho}(X, \partial X)+\tau_{\rho}(\partial X)
$$

\section{Analytic torsion of infinite group actions}

If one has a compact Lie group acting on a closed oriented manifold $X$, one may try to define an analytic torsion as in $\S$ II. That is, if $\rho: G \rightarrow O(N)$ is an orthogonal representation of $G$, then let

$$
\Pi=\frac{1}{\operatorname{Vol}(G)} \int_{G} \rho(g) a\left(g^{-1}\right)^{*} d g
$$

denote the projection onto $\rho$-invariant $\mathbb{R}^{N}$-valued differential forms, and put

$$
T_{\rho}=\left.\frac{d}{d s}\right|_{s=0} \frac{1}{\Gamma(s)} \int_{0}^{\infty} T^{s-1} \operatorname{Tr}(-)^{F} F \Pi e^{-T \Delta^{\prime}} d T
$$


The problem is that it is easy to see that even when $\rho$ gives an acyclic complex of equivariant differential forms, the torsion $T_{\rho}$ may depend on the $G$-invariant Riemannian metric used in its definition.

Example. Consider the metric on $S^{1}$ with total length $2 \pi R$, the $U(1)$ action on $S^{1}$ given by $a\left(e^{i \theta}\right)\left(e^{i \varphi}\right)=e^{i(\theta+\varphi)}$, and the character $c\left(e^{i \theta}\right)=$ $2 \cos (k \theta), k \neq 0$. Then the Laplacian acting on equivariant 1 -forms has only $k^{2} / R^{2}$ as an eigenvalue (with multiplicity two), and so $T$ will have a term proportionate to $\ln R$.

In terms of the proof of invariance for finite group actions, which was used in Proposition 2, what goes wrong is that for the map $\Phi: G \times X \rightarrow$ $G \times X$ given by $\Phi(g, x)=(g, g x)$ we need to have a fixed point set which is a smooth submanifold of $G \times X$ of even codimension. In general, the fixed point set of $\Phi$ will have singularities [3].

In order to define an invariant for the case of infinite group actions, let us forget any smooth or continuous structure that $G$ may have, and simply consider the case of a discrete group $G$ acting by smooth orientationpreserving diffeomorphisms on $X$ such that closure of $G$ in $\operatorname{Diff}(X)$ is compact, i.e., there are metrics on $X$ so that $G$ acts by isometries. We can define a function on $G$ by

$$
t(g)=\left.\frac{d}{d s}\right|_{s=0} \frac{1}{\Gamma(s)} \int_{0}^{\infty} T^{s-1} \operatorname{Tr}(-)^{F} F a\left(g^{-1}\right)^{*} e^{-T \Delta^{\prime}},
$$

where $\Delta^{\prime}$ acts on $\Lambda^{*}(X)$. Under a change of the $G$-invariant metric, the proof of Proposition 2 shows that

$$
\frac{d}{d \varepsilon} g(t)=-\left.\operatorname{Tr}\right|_{\operatorname{Ker} \Delta}(-)^{F}\left(\frac{d}{d \varepsilon} *\right) *^{-1} a\left(g^{-1}\right)^{*} .
$$

Let $f$ be a complex function on $G$, which is zero on all but a finite number of group elements, and consider

$$
C_{f} \equiv \sum_{g} f(g) t(g) \text {. }
$$

Then

$$
\frac{d}{d \varepsilon} C_{f}=-\left.\operatorname{Tr}\right|_{\operatorname{Ker} \Delta}(-)^{F}\left(\frac{d}{d \varepsilon} *\right) *^{-1} \sum_{g} f(g) a\left(g^{-1}\right)^{*} .
$$

Now $a\left(g^{-1}\right)^{*}$ commutes with the de Rham isomorphism from $\operatorname{Ker} \Delta$ to $H^{*}(X)$, and so $\sum_{g} f(g) a\left(g^{-1}\right)^{*}$ is zero on $\operatorname{Ker} \Delta$ if and only if it is zero on $H^{*}(X)$. Thus for all $f$ such that $\sum_{g} f(g) a\left(g^{-1}\right)^{*}$ vanishes on $H^{*}(X), C_{f}$ is metric independent. 
In other words, we have constructed a smooth invariant $I$ which lies in the dual space to

$$
\begin{aligned}
& \mathscr{F}^{(G)}=\left\{f \in \mathbb{C}^{G}:\right. f \text { has finite support and } \\
&\left.\qquad \sum_{g} f(g) a\left(g^{-1}\right)^{*} \text { vanishes on } H^{*}(X)\right\} .
\end{aligned}
$$

$I$ is defined by

$$
\langle I, f\rangle=\sum_{g} f(g) t(g) .
$$

If $G$ happens to be a connected Lie group acting smoothly on $X$, then for all $g \in G, a\left(g^{-1}\right)^{*}$ is the identity on $H^{*}(X)$ and so $\mathscr{F}^{(G)}=\left\{f \in \mathbb{C}^{G}: f\right.$ has finite support and $\left.\sum_{g} f(g)=0\right\}$. We can identify $\mathscr{F}^{(G)^{*}}$ as

$$
\mathscr{F}^{(G)^{*}} \approx\left\{h \in \mathbb{C}^{G}: h(e)=0\right\}
$$

via the pairing $\langle h, f\rangle=\sum_{g} h(g) f(g)$.

We have the following analogues of the propositions of $\S \S I$ II and III:

Proposition 27. If $X$ has even dimension, then $C_{f}=0$.

Proposition 28. Suppose that $G_{1}$ acts on $X_{1}$ by orientation-preserving isometries and that $G_{2}$ acts on $X_{2}$ by orientation-preserving isometries. Then for $f_{1} \in \mathscr{F}\left(G_{1}\right)$ and $f_{2} \in \mathscr{F}\left(G_{2}\right)$,

$$
C_{f_{1} \otimes f_{2}}=C_{f_{1}} L_{f_{2}}+C_{f_{2}} L_{f_{1}}
$$

where

$$
L_{f}=\sum_{g} f(g) L(g),
$$

and $L(g)$ denotes the Lefschetz number of the g-action.

Proposition 29. Suppose that $G_{1} \subset G_{2}$ and that $G_{2}$ acts on $X$ by orientation-preserving isometries. Let $i$ be the inclusion of $\mathscr{F}\left(G_{1}\right)$ into $\mathscr{F}\left(G_{2}\right)$. Then for all $f \in \mathscr{F}\left(G_{1}\right), t_{f}=t_{i(f)}$.

Proposition 30. Given $\gamma \in \mathscr{F}(G)$, define $f^{\prime}(g)=f\left(\gamma g \gamma^{-1}\right)$. Then $t_{f^{\prime}}=t_{f}$.

\section{Computations of $I$}

We will need:

Proposition 31. For $\alpha \in[0,1)$, put $L(\alpha, s)=\sum_{n \neq 0}\left(n^{2}\right)^{-s} e^{2 \pi i \alpha n}$. Then 
(11.1) (i) $L(\alpha, 0)=-1$,

(11.2) (ii)

$$
\left.\frac{d}{d s}\right|_{s=0} L(\alpha, s)=\left\{\begin{array}{l}
-2 \ln 2 \pi \quad \text { if } \alpha=0, \\
-(2 \ln 2 \pi+2 \gamma+\Psi(\alpha)+\psi(1-\alpha)) \quad \text { if } \alpha \neq 0,
\end{array}\right.
$$

where $\gamma$ is the Euler-Mascheroni constant, and $\psi(\alpha)=\Gamma^{\prime}(\alpha) / \Gamma(\alpha)$.

Proof. For $\alpha=0, L(0, s)=2 \zeta(2 s)$ and so

$$
L(0,0)=-1,\left.\quad \frac{d}{d s}\right|_{s=0} L(0, s)=-4 \zeta^{\prime}(0)=-2 \ln 2 \pi .
$$

For $\alpha \neq 0$, put

$$
\Phi(\alpha, a, s)=\sum_{n=0}^{\infty} e^{2 \pi i n \alpha} /(n+a)^{s}
$$

Then

$$
L(\alpha, s)=e^{2 \pi i \alpha} \Phi(\alpha, 1,2 s)+e^{2 \pi(1-\alpha)} \Phi(1-\alpha, 1,2 s) .
$$

By the well-known Lerch relation,

$$
\begin{aligned}
& \Phi(\alpha, 1,1-s) \\
& \quad=\Gamma(s)(2 \pi)^{-s}\left[e^{\pi i(s / 2-2 \alpha)} \zeta(s, \alpha)+e^{\pi i(-s / 2-2 \alpha)} \zeta(s, 1-\alpha)\right] .
\end{aligned}
$$

(The statement of this is essentially exercise 8 on p. 280 of [21]. However, as $a<1$ in the exercise, it does not cover the range of variables that we need. One can prove the needed identity directly by forming the contour integral as in 13.15 of [21] and adding the residues of the poles.)

Thus,

$$
\begin{aligned}
L(\alpha, s)=(2 \pi)^{2 s-1} \Gamma(1-2 s) i\left[e^{-\pi i s} \zeta(1-2 s, \alpha)\right. & \\
-e^{-\pi i s} \zeta(1-2 s, 1-\alpha)+e^{-\pi i s} \zeta(1-2 s, 1-\alpha) & \left.-e^{\pi i s} \zeta(1-2 s, \alpha)\right] \\
=2(2 \pi)^{2 s-1} \Gamma(1-2 s)(\sin \pi s)[\zeta(1-2 s, \alpha) & +\zeta(1-2 s, 1-\alpha)] .
\end{aligned}
$$

Because

$$
\zeta(z, v)=(z-1)^{-1}-\psi(v)+o(z-1),
$$

and

$$
\Gamma(z)=1-\gamma(z-1)+o(z-1)^{2},
$$


we have that

$$
L(\alpha, s)=-1-(2 \ln 2 \pi+2 \gamma+\psi(\alpha)+\psi(1-\alpha)) s+O\left(s^{2}\right) .
$$

Example 1. $\mathbb{Z}$ acts on $S^{1}$. For $n \in \mathbb{Z}$, suppose that the action of $n$ on $S^{1}$ is given by

$$
a(n)\left(e^{i \theta}\right)=e^{i(\theta+2 \pi n \alpha)}, \quad 0 \leq \alpha<1 .
$$

Using the standard metric on $S^{1}$, we obtain

$$
\operatorname{Tr} a(-n)^{*}\left(\Delta^{\prime}\right)^{-s}\left(\text { acting on } \Lambda^{1}\left(S^{1}\right)\right)=L(n \alpha-[n \alpha], s)
$$

and

$$
\begin{aligned}
t(n) & =-\left.\frac{d}{d s}\right|_{s=0} \operatorname{Tr} a(-n)^{*}\left(\Delta^{\prime}\right)^{-s} \\
& =-\left.\frac{d}{d s}\right|_{s=0} L(n \alpha-[n \alpha], s) \\
& =\left\{\begin{array}{r}
2 \ln 2 \pi \text { if } n \alpha \in \mathbb{Z}, \\
2 \ln 2 \pi+2 \gamma+\psi(n \alpha-[n \alpha]) \\
\quad+\psi(1-n \alpha+[n \alpha]) \text { if } n \alpha \notin \mathbb{Z} .
\end{array}\right.
\end{aligned}
$$

Thus,

$$
I(n)=\left\{\begin{array}{l}
0 \text { if } n \alpha \in \mathbb{Z}, \\
2 \gamma+\psi(n \alpha-[n \alpha])+\psi(1-n \alpha+[n \alpha]) \quad \text { if } n \alpha \notin \mathbb{Z} .
\end{array}\right.
$$

Because any orientation-preserving diffeomorphism of $S^{1}$, which preserves some metric, is conjugate to a rotation, Proposition 32 will show that the invariant $I$ classifies such a diffeomorphism up to conjugacy.

Example 2. $\mathbb{Z}$ acts on a sphere by special orthogonal transformations. If the sphere is even-dimensional, then by Proposition 1, $T$ vanishes. Assume that $\mathbb{Z}$ acts on $S^{2 N-1}$ specially orthogonally. By putting the action into normal form, we can assume that $n \in \mathbb{Z}$ acts by

$$
a(n)\left(\left\{z_{j}\right\}_{j=1}^{N}\right)=\left\{e^{2 \pi i n \alpha(j)} z_{j}\right\}_{j=1}^{N},
$$

where $\sum_{j=1}^{N}\left|z_{j}\right|^{2}=1$, and each $\alpha(j)$ lies in $[0,1)$. From the results of Ray [16], it follows that if $\sum f(n)=0$, then

$$
\Sigma f(n) \operatorname{Tr} a(-n)^{*}(-)^{F} F\left(\Delta^{\prime}\right)^{-s}=-\sum_{j=1}^{N} L(\alpha(j), s) .
$$


Thus,

$$
I(n)=\sum_{j=1}^{N}\left\{\begin{array}{l}
0 \quad \text { if } n \alpha(j) \in \mathbb{Z}, \\
2 \gamma+\psi(n \alpha(j)-[n \alpha(j)]) \\
\quad+\psi(1-n \alpha(j)+[n \alpha(j)]) \quad \text { if } n \alpha(j) \notin \mathbb{Z} .
\end{array}\right.
$$

Proposition 32. Two special orthogonal actions on $S^{2 N-1}$ are smoothly equivalent if and only if they are linearly equivalent.

Note. This theorem is due to de Rham, who proved it using the ordinary Reidemeister torsion, along with a geometric argument [18]. We will prove it analytically, using the invariant I.)

For the proof of the proposition, we must first state and prove the following.

Lemma. The function

$$
f(z)=2 \gamma+\psi(z)+\psi(1-z)
$$

is monotonically increasing on the interval $\left(0, \frac{1}{2}\right)$.

Proof. We have that

$$
\psi(z)=-\gamma+\sum_{n=1}^{\infty}\left[n^{-1}-(n+z-1)^{-1}\right]
$$

[7], and so

$$
\psi^{\prime}(z)=\sum_{n=1}^{\infty}\left[(n+z-1)^{-2}\right]
$$

and

$$
f^{\prime}(z)=\psi^{\prime}(z)-\psi^{\prime}(1-z)=\sum_{n=1}^{\infty}\left[(n-(1-z))^{-2}-(n-z)^{-2}\right]
$$

But for $z \in\left(0, \frac{1}{2}\right)$ and $n \in \mathbb{Z}^{+},(n-z)^{-2}<(n-(1-z))^{-2}$ and hence the lemma follows.

Proof of Proposition 32. Note that $f\left(\frac{1}{2}\right)=2 \gamma+2 \psi\left(\frac{1}{2}\right)=-4 \ln 2$. Thus we can immediately determine the number of irrational $\alpha(j)$ 's for a given rotation by computing irr $=\left(\sup _{n \in \mathbb{Z}} I(n)\right) /(-4 \ln 2)$. If irr $>0$, let us define a function on the torus $T^{\mathrm{irr}}$ by

$$
g\left(\left\{e^{2 \pi i \theta(j)}\right\}\right)=\sum_{j=1}^{\text {irr }}\left\{\begin{aligned}
-\infty & \text { if } \theta(j) \in \mathbb{Z}, \\
2 \gamma & +\psi(\theta(j)-[\theta(j)]) \\
& +\psi(1-\theta(j)+[\theta(j)]) \text { if } \theta(j) \notin \mathbb{Z} .
\end{aligned}\right.
$$


Note that $\sup g=-4$ irr $\ln 2<-2$. For any real $\varepsilon$, define a function $g_{\varepsilon}$ on $T^{\text {irr }}$ by

$$
g_{\varepsilon}\left(\left\{e^{2 \pi i \theta(j)}\right\}\right)=\max \left(g\left(\left\{e^{2 \pi i \theta(j)}\right\}\right), \varepsilon\right) .
$$

Choose $\varepsilon$ so that the average value of $g$ on $T^{\mathrm{irr}}$ is -2 . (By the above bound on $\sup g$, this can be done.) For any integer $h>0$, compute

$$
\operatorname{av}(h)=\lim _{M \rightarrow \infty} M^{-1} \sum_{n=1}^{M} \max (I(h n), \varepsilon) .
$$

By the uniform distribution of an irrational angle flow on a torus, we know that $\sup _{h} \operatorname{av}(h)=-2$ irr. If irr $\neq N$, then there will be a smallest $h$ such that $\operatorname{av}(h)=-2 \mathrm{irr}$, and this $h$ will be the order of the cyclic group generated by the rational part of the rotation. Thus this order, which we will denote by $p$, is determined by the invariant $I$.

Let us now consider two rotations with the same number irr of irrational $\alpha(j)$ 's and the same order $p$ of the finite part. We wish to show that the invariant $I$ determines the irrational $\alpha(j)$ 's up to order and signs. For a given rotation, define $J(n)=I(p n)$. If $R$ denotes the transformation on $T^{\mathrm{irr}}$ which is a translation by the angles $2 \pi p \alpha(j)$, then $J(n)=g\left(R^{n}\{1\}\right)$. Thus it suffices to show that if we have two transformations $R_{1}$ and $R_{2}$ of $T^{\mathrm{irr}}$ which are both translations by irrational angles, and $g\left(R_{1}^{n}(\{1\})\right)=g\left(R_{2}^{n}(\{1\})\right)$ for all $n$, then $R_{1}$ and $R_{2}$ are conjugate by the action on $T^{\text {irr }}$ of a matrix in $\mathrm{O}(\mathrm{irr}, \mathbb{Z})$.

Let $U_{\delta} \subset T^{\text {irr }}$ denote $g^{-1}(-\infty, \delta)$. Then the $U_{\delta}$ 's form decreasing neighborhoods of the point $\{1\} \in T^{\text {irr }}$. Let $z_{1}=R_{1}(\{1\})$ be thought of as an element of the group $T^{\mathrm{irr}}$, and similarly for $z_{2}=R_{2}(\{1\})$. Then for any integer $m, z_{1}^{m} \in U_{\delta}$ if and only if $z_{2}^{m} \in U_{\delta}$. Consider the map $\varphi$ which sends $z_{1}^{m}$ to $z_{2}^{m}$ for all $m \in \mathbb{Z}$. As $\left\{z_{1}^{m}\right\}$ is dense in $T^{\text {irr }}$, and $\varphi$ is uniformly continuous, it follows that $\varphi$ extends to a homomorphism from $T^{\mathrm{irr}}$ to $T^{\mathrm{irr}}$, and so $\varphi$ is induced from the action of an element $M$ of $\operatorname{gl}($ irr, $\mathbb{Z})$ on $\mathbb{R}^{\text {irr }}$. Furthermore, for all $\delta, \varphi\left(U_{\delta}\right)=U_{\delta}$. By taking $\delta$ sufficiently small, we see that $M$ must preserve the $L^{1}$ norm on $\mathbb{R}^{\text {irr }}$, and so must lie in $\mathrm{O}($ irr, $\mathbb{Z})$. Thus the invariant $I$ determines the irrational part of a rotation uniquely, up to order and signs.

We can now subtract off the contributions to $I$ of the irrational rotations to obtain the invariant for a cyclic action of order $p$. Suppose that we have two such actions, which are rotations by angles $\left\{2 \pi \nu_{1}(l) / p\right\}_{l=1}^{n}$ and $\left\{2 \pi \nu_{2}(l) / p\right\}_{l=1}^{n}$ respectively. Then by Proposition 7 , we know that 
the discrete Fourier transform of $I$ is given by

$$
F_{k}=\sum_{\substack{l \\ \operatorname{gcd}(l) \mid k}} \log \left|-e^{2 \pi i k \alpha(l) / p}\right|,
$$

provided that $p \nmid k$. Here $\operatorname{gcd}(l)=\operatorname{gcd}\left(\nu_{l}, p\right)$, and $\alpha(l)$ is defined by

$$
\alpha(l) \frac{\nu_{l}}{\operatorname{gcd}\left(\nu_{l}, p\right)} \equiv 1 \quad \bmod \frac{p}{\operatorname{gcd}\left(\nu_{l}, p\right)} .
$$

We want to show that if two rotations have the same such transform, then their indices $\left\{\nu_{1}(l)\right\}_{l=1}^{n}$ and $\left\{\nu_{2}(l)\right\}_{l=1}^{n}$ coincide up to order and signs.

By induction, we may assume that this is true for the actions of all proper subgroups of $Z_{p}$. Because the transform on a proper subgroup of $Z_{p}$ is determined by the transform on $Z_{p}$, we may assume that our two rotations have the same $\nu_{l}$ 's which have a nontrivial common divisor with $p$, up to order and signs. We can then subtract the contribution of these $\nu_{l}$ 's from $\tau$, and hereafter assume that for all $l, \operatorname{gcd}(l)=1$.

We now have two rotations such that

$$
F_{k_{1}}=\sum_{l} \log \left|1-e^{2 \pi i k \alpha_{1}(l) / p}\right|
$$

and

$$
F_{k_{2}}=\sum_{l} \log \left|1-e^{2 \pi i k \alpha_{2}(l) / p}\right|
$$

coincide whenever $p \nmid k$. Furthermore, we have that $\operatorname{gcd}\left(\alpha_{1}(l), p\right)=$ $\operatorname{gcd}\left(\alpha_{2}(l), p\right)=1$ for each $l$. It follows from Franz's lemma [14] that the indices $\left\{\alpha_{1}(l)\right\}$ and $\left\{\alpha_{2}(l)\right\}$ coincide up to order and signs. Thus the indices $\left\{\nu_{1}(l)\right\}$ and $\left\{\nu_{2}(l)\right\}$ coincide up to order and signs.

\section{Bibliography}

[1] M. Berger, P. Gauduchon \& E. Mazet, Le spectre d'une variete Riemannienne, Springer, Berlin, 1971.

[2] G. Bredon, Introduction to compact transformation groups, Academic Press, New York, 1972.

[3] J. Brüning \& E. Heintze, The asymptotic expansion of Minakshisundaram-Pleijel in the equivariant case, Duke Math. J. 51 (1984) 959-980.

[4] J. Cheeger, Analytic torsion and the heat equation, Ann. of Math. 109 (1979) 259-321.

[5] _ , preprint version of [4], unpublished.

[6] S. Della Pietra \& V. Della Pietra, Analytic torsion and finite group actions, Inst. for Advanced Study, preprint, 1988.

[7] A. Erdelyi, Higher transcendental functions, the Bateman manuscript project, Vol. 1, McGraw-Hill, New York, 1953.

[8] D. Fried, Lefschetz formulas for flows, Contemp. Math. 58 III, Lefschetz Centennial Conference, 1987, 19-69. 
[9] P. Gilkey, Invariance theory, the heat equation and the Atiyah-Singer index theorem, Publish or Perish, Boston, 1984.

[10] M. Hirsch, Differential topology, Springer, New York, 1976.

[11] S. Lang, Algebra, Addison-Wesley, Reading, MA, 1971.

[12] J. Lott, Analytic torsion for group actions, Contemp. Math., Proc. Amer. Math. Soc. Special Session in Differential Geometry, Los Angeles, 1987 (to appear).

[13] W. Lück, Analytic and topological torsion for manifolds with boundary (to appear).

[14] J. Milnor, Whitehead torsion, Bull. Amer. Math. Soc. 72 (1966), 358-426.

[15] W. Müller, Analytic torsion and R-torsion of Riemannian manifolds, Advances in Math. 28 (1978) 233-305.

[16] D. B. Ray, Reidemeister torsion and the Laplacian on lens spaces, Advances in Math. 4 (1970) 109-126.

[17] D. B. Ray \& I. M. Singer, R-torsion and the Laplacian on Riemannian manifolds, Advances in Math. 7 (1971) 145-210.

[18] G. de Rham, Reidemeister's torsion invariant and rotations of $S^{n}$, Differential Analysis, Bombay Colloquium, Oxford Univ. Press, 1964.

[19] M. Rothenberg, Torsion invariants and finite transformation groups, Proc. Sympos. Pure Math., Vol. 32, Amer. Math. Soc., Providence, RI, 1978.

[20] A. Wasserman, Equivariant differential topology, Topology 8 (1969) 127-150.

[21] E. Whittaker \& G. Watson, $A$ course of modern analysis, Cambridge Univ. Press, 1915.

UNIVERSITY OF MICHIGAN UNIVERSITY OF CHICAGO 
